Publ. RIMS, Kyoto Univ.

15 (1979), 469-518

\title{
On a Sufficient Condition for Well-posedness of Weakly Hyperbolic Cauchy Problems
}

\author{
By
}

Reiko SAKAMOTO*

Already, we have good results on this theme for 2-nd order equations ([1]). Recently, we have also many results for higher order equations with smooth characteristic roots ([2], [3]).

In this paper, we consider only weakly hyperbolic equations with double characteristic roots at most, which may be non-smooth.

\section{Chapter $\mathbb{1}$. Energy Inequalities}

\section{$\S \mathbb{1}$. Condition (A)}

Let us consider the Cauchy problem:

$$
\left\{\begin{array}{l}
A\left(t, x^{\prime} ; D_{t}, D_{x}^{\prime}\right) u=f\left(t, x^{\prime}\right) \quad \text { in }\left\{t>0, x^{\prime} \in \mathbb{R}^{n}\right\}, \\
\left.D_{t}^{j} u\right|_{t=0}=u_{j}\left(x^{\prime}\right) \quad(j=0,1, \cdots, m-1) \quad \text { in }\left\{x^{\prime} \in \mathbb{R}^{n}\right\},
\end{array}\right.
$$

where $D_{t}=\frac{1}{i} \frac{\partial}{\partial t}, \quad D_{x}^{\prime}=\left(\frac{1}{i} \frac{\partial}{\partial x_{1}}, \cdots, \frac{1}{i} \frac{\partial}{\partial x_{n}}\right)$ and

$$
A\left(t, x^{\prime} ; \tau, \xi^{\prime}\right)=\sum_{i+|\nu| \leq m} a_{i \nu}\left(t, x^{\prime}\right) \tau^{i \xi^{\prime \nu}} .
$$

Now we denote

$$
\begin{aligned}
& A_{0}\left(t, x^{\prime} ; \tau, \xi^{\prime}\right)=\sum_{i+\mid \nu i=m} a_{i \nu}\left(t, x^{\prime}\right) \tau^{i \xi^{\prime \nu}}, \\
& A_{1}\left(t, x^{\prime} ; \tau, \xi^{\prime}\right)=\sum_{i+|\nu|=m-1} a_{i \nu}\left(t, x^{\prime}\right) \tau^{i} \xi^{\prime \nu},
\end{aligned}
$$

then our consideration will be based on the assumption on $A_{0}$ :

Communicated by S. Matsuura, April 16, 1977.

* Department of Mathematics, Nara Women's University. 
Condition (A).

(A. 1) $a_{i v}\left(t, x^{\prime}\right)$ are smooth and constant outside a ball in $\boldsymbol{R}^{n+1}$.

(A. 2) $A_{0}$ is hyperbolic with respect to $\tau$, that is,

$$
\left\{\begin{array}{l}
A_{0}\left(t, x^{\prime} ; 1,0\right) \neq 0, \\
A_{0}\left(t, x^{\prime} ; \tau^{\prime}, \xi^{\prime}\right) \neq 0 \text { for } \operatorname{Im} \tau \neq 0, \xi^{\prime} \in \boldsymbol{R}^{n} .
\end{array}\right.
$$

(A. 3) Roots of $A_{0}\left(t, x^{\prime} ; \tau, \xi^{\prime}\right)=0$ reith respect to $\tau$ are at most clouble for $\left(t, x^{\prime}, \xi^{\prime}\right) \in \boldsymbol{R}^{n+1} \times\left(\boldsymbol{R}^{n}-\{0\}\right)$.

For convenience, we denote

$$
\begin{aligned}
& x=\left(t, x^{\prime}\right)=\left(x_{0}, x_{1}, \cdots, x_{n}\right), \xi=\left(\tau, \xi^{\prime}\right)=\left(\xi_{0}, \xi_{1}, \cdots, \xi_{n}\right), \\
& X^{\prime}=\left(x, \xi^{\prime}\right) \in \boldsymbol{R}^{n+1} \times\left(\boldsymbol{R}^{n}-\{0\}\right), \quad X=\left(\tau, X^{\prime}\right),
\end{aligned}
$$

and we assume $A_{0}(x ; 1,0)=1$. We denote

$$
A_{0}(X)=\prod_{i=1}^{m}\left(\tau-\tau_{i}\left(X^{\prime}\right)\right)
$$

In the following, let us consider the behaviour of characteristic roots $\tau_{i}\left(X^{\prime}\right)$ at $X^{\prime}=X_{0}^{\prime}$ and in its small neighbourhood $U$. We may assume

$$
\tau_{1}\left(X_{0}^{\prime}\right), \tau_{2}\left(X_{0}^{\prime}\right), \cdots, \tau_{m-d}\left(X_{0}^{\prime}\right)
$$

are distinct each other, and

$$
\tau_{m-d+1}\left(X_{0}^{\prime}\right)=\tau_{1}\left(X_{0}^{\prime}\right), \cdots, \tau_{m}\left(X_{0}^{\prime}\right)=\tau_{d}\left(X_{0}^{\prime}\right) .
$$

Then, there exists a neighbourhood $U$ of $X^{\prime}=X_{0}^{\prime}$, where

$$
\begin{aligned}
H_{i}(X) & =\left(\tau-\tau_{i}\left(X^{\prime}\right)\right)\left(\tau-\tau_{m-d+i}\left(X^{\prime}\right)\right) \\
& =\left(\tau-\alpha_{i}\left(X^{\prime}\right)\right)^{2}-\beta_{i}\left(X^{\prime}\right)=h_{i}(X)^{2}-\beta_{i}\left(X^{\prime}\right) \quad(i=1, \cdots, d),
\end{aligned}
$$

where $\alpha_{i}\left(X^{\prime}\right)$ and $\beta_{i}\left(X^{\prime}\right)$ are real-valued smooth function and $\beta_{i}\left(X^{\prime}\right) \geq 0$,

(ii) $\tau_{i}\left(X^{\prime}\right)(i=d+1, \cdots, m-d)$ are real-valued smooth functions.

Now, we denote

$$
h_{i}(X)=\tau-\tau_{i}\left(X^{\prime}\right)=\tau-\alpha_{i}\left(X^{\prime}\right) \quad(i=d+1, \cdots, m-d),
$$

then

$$
\left\{\alpha_{1}\left(X^{\prime}\right), \cdots, \alpha_{m-d}\left(X^{\prime}\right)\right\}
$$

are distinct, real-valued and smooth functions in $U$. Hence, we denote 


$$
\left\{\begin{array}{l}
P_{i}(X)=h_{i}(X) \frac{A_{0}(X)}{H_{i}(X)} \quad(i=1, \cdots, d), \\
P_{i}^{\prime}(X)=\frac{A_{0}(X)}{H_{i}(X)}=\frac{P_{i}(X)}{h_{i}(X)} \quad(i=1, \cdots, d), \\
P_{i}(X)=\frac{A_{0}(X)}{h_{i}(X)} \quad(i=d+1, \cdots, m-d),
\end{array}\right.
$$

then these $m$ polynomials with respect to $\tau$ with smooth coefficients defined in $U$ make a base of the linear space of polynomials of order less than $m$ with respect to $\tau$.

Now we proceed to the next step, assuming $U=\mathbb{R}^{n+1} \times\left(\mathbb{R}^{n}-\{0\}\right)$, which will be justified in Section 5 . Then we have smooth decompositions of $A_{0}(X)$ :

$$
\left\{\begin{array}{l}
A_{0}(X)=H_{i}(X) P_{i}^{\prime}(X) \quad(i=1, \cdots, d), \\
A_{0}(X)=h_{i}(X) P_{i}(X) \quad(i=d+1, \cdots, m-d)
\end{array}\right.
$$

for $X^{\prime} \in \mathbb{R}^{n+1} \times\left(\mathbb{R}^{n}-\{0\}\right)$, therefore we have decompositions of $A(D)$ $=A(x, D)$ :

\section{Leimma 1.1.}

$$
A(D)=\widetilde{H}_{i}(D) \widetilde{P}_{i}^{\prime}(D)+\{\{m-2\}\} \quad(i=1, \cdots, d),
$$

where

$$
\begin{aligned}
& \tilde{H}_{i}(D)=\left(D_{t}-\widetilde{\alpha}_{i}\left(D^{\prime}\right)\right)^{2}-\tilde{\beta}_{i}\left(D^{\prime}\right)=\tilde{h}_{i}(D)^{2}-\tilde{\beta}_{i}\left(D^{\prime}\right), \\
& \widetilde{\alpha}_{i}\left(D^{\prime}\right)=\alpha_{i}\left(D^{\prime}\right)+\alpha_{i}^{1}\left(D^{\prime}\right) \quad\left(\alpha_{i}^{1}\left(X^{\prime}\right) \text { : homogeneous of degree } 0\right), \\
& \tilde{\beta}_{i}\left(D^{\prime}\right)=\beta_{i}\left(D^{\prime}\right)+\beta_{i}^{1}\left(D^{\prime}\right) \quad\left(\beta_{i}^{1}\left(X^{\prime}\right): \text { homogeneous of degree } 1\right), \\
& \widetilde{P}_{i}^{\prime}(D)=P_{i}^{\prime}(D)+P_{i}^{\prime 1}(D) \quad\left(P_{1}^{\prime 1}(X) \text { : homogeneous of degree } m-3\right) .
\end{aligned}
$$

(ii) $\quad A(D)=\tilde{h}_{i}(D) \widetilde{P}_{i}(D)+\{\{m-2\}\} \quad(i=d+1, \cdots, m-d)$,

zehere

$$
\begin{aligned}
& \tilde{h}_{i}(D)=D_{t-} \tilde{\alpha}_{i}\left(D^{\prime}\right), \\
& \widetilde{\alpha}_{i}\left(D^{\prime}\right)=\alpha_{i}\left(D^{\prime}\right)+\alpha_{i}^{1}\left(D^{\prime}\right) \quad\left(\alpha_{i}^{1}\left(X^{\prime}\right) \text { : homogeneous of degree } 0\right) \text {, } \\
& \widetilde{P}_{i}(D)=P_{i}(D)+P_{i}^{1}(D) \quad\left(P_{i}^{1}(X) \text { : homogeneous of degree } m-2\right) \text {. }
\end{aligned}
$$


Notation 1. $\{\{m-2\}\}$ means an operator of order not greater than $m-2$.

Notation 2. Homogeneous of degree $k$ means homogeneous of degree $k$ with respect to $\xi=\left(\tau, \xi^{\prime}\right)$, which is a polynomial of degree not greater than $k$ with respect to $\tau$.

Proof of (i). Let $a(D) \circ b(D)$ be a singular integral operator with simbol $a(X) b(X)$, then we have

$$
\begin{aligned}
A_{0}(D) & =H_{i}(D) \circ P_{i}^{\prime}(D) \\
& =H_{i}(D) P_{i}^{\prime}(D)-\left(D_{\xi} H_{i}\right)(D) \circ\left(\partial_{x} P_{i}^{\prime}\right)(D)+\{\{m-2\}\},
\end{aligned}
$$

where

$$
\begin{aligned}
& \left(D_{\xi} H_{i}\right)(X)\left(\partial_{x} P_{i}^{\prime}\right)(X)=\sum_{j=0}^{n}\left(D_{\xi j} H_{i}\right)(X)\left(\partial_{x_{j}} P_{i}^{\prime}\right)(X), \\
& \partial_{t}=\frac{\partial}{\partial t}, \quad \partial_{x_{j}}=\frac{\partial}{\partial x_{j}} .
\end{aligned}
$$

Therefore we have

$$
A(D)=H_{i}(D) P_{i}^{\prime}(D)+R_{i}(D)+\{\{m-2\}\},
$$

where

$$
R_{i}(X)=A_{1}(X)-\left(D_{\xi} H_{i}\right)(X)\left(\partial_{x} P_{i}^{\prime}\right)(X)
$$

Using the representation of $R_{i}(X)$ by $\left\{P_{j}^{\prime}(X) \quad(j=1, \cdots, d), P_{j}(X)\right.$ $(j=1, \cdots, m-d)\}$, we have

$$
R_{i}(X)=c_{i}\left(X^{\prime}\right) P_{i}(X)+d_{i}\left(X^{\prime}\right) P_{i}^{\prime}(X)+S_{i}(X) H_{i}(X),
$$

where $c_{i}\left(X^{\prime}\right), d_{i}\left(X^{\prime}\right), S_{i}(X)$ are smooth and homogeneous. Hence we have

$$
\begin{aligned}
& A(D)=\left\{H_{i}(D)+\right.\left.c_{i}\left(D^{\prime}\right) \circ h_{i}(D)+d_{i}\left(D^{\prime}\right)\right\} \\
& \times\left\{P_{i}^{\prime}(D)+S_{i}(D)\right\}+\{\{m-2\}\} \\
&=\left\{h_{i}(D) \circ h_{i}(D)-\beta_{i}\left(D^{\prime}\right)+c_{i}\left(D^{\prime}\right) \circ h_{i}(D)+d_{i}\left(D^{\prime}\right)\right\} \\
& \times \\
& \times\left\{P_{i}^{\prime}(D)+S_{i}(D)\right\}+\{\{m-2\}\}
\end{aligned}
$$




$$
\begin{aligned}
= & \left\{\left(h_{i}(D)+\frac{1}{2} c_{i}\left(D^{\prime}\right)\right)^{2}-\left(\beta_{i}\left(D^{\prime}\right)-d_{i}\left(D^{\prime}\right)\right.\right. \\
& \left.\left.+\left(D_{\xi} h_{i}\right)\left(D^{\prime}\right) \circ\left(\partial_{x} h_{i}\right)\left(D^{\prime}\right)\right)\right\}\left\{P_{i}^{\prime}(D)+S_{i}(D)\right\}+\{\{m-2\}\} \\
= & \widetilde{H}_{i}(D) \widetilde{P}_{i}^{\prime}(D)+\{\{m-2\}\},
\end{aligned}
$$

where

$$
\begin{aligned}
& \widetilde{H}_{i}(D)=\widetilde{h}_{i}(D)^{2}-\tilde{\beta}_{i}\left(D^{\prime}\right), \quad h_{i}(D)=D_{t}-\widetilde{\alpha}_{i}\left(D^{\prime}\right), \\
& \widetilde{\alpha}_{i}\left(D^{\prime}\right)=\alpha_{i}\left(D^{\prime}\right)-\frac{1}{2} c_{i}\left(D^{\prime}\right), \\
& \tilde{\beta}_{i}\left(D^{\prime}\right)=\beta_{i}\left(D^{\prime}\right)-d_{i}\left(D^{\prime}\right)+\left(D_{\xi} h_{i}\right)\left(D^{\prime}\right) \circ\left(\partial_{x} h_{i}\right)\left(D^{\prime}\right), \\
& \widetilde{P}_{i}^{\prime}(D)=P_{i}^{\prime}(D)+S_{i}(D) .
\end{aligned}
$$

Proof of (ii). Since

$$
\begin{aligned}
A_{0}(D) & =h_{i}(D) \circ P_{i}(D) \\
& =h_{i}(D) P_{i}(D)-\left(D_{\xi} h_{i}\right)\left(D^{\prime}\right) \circ\left(\partial_{x} P_{i}\right)(D)+\{\{m-2\}\},
\end{aligned}
$$

we have

$$
A(D)=h_{i}(D) P_{i}(D)+R_{i}(D)+\{\{m-2\}\},
$$

where

$$
R_{i}(X)=A_{1}(X)-\left(D_{\xi} h_{i}\right)(X)\left(\partial_{x} P_{i}\right)(X) .
$$

Using the representation of $R_{i}(X)$ by $\left\{P_{j}^{\prime}(j=1, \cdots, d), P_{j}(j=1, \cdots\right.$, $m-d)\}$, we have

$$
R_{i}(X)=c_{i}\left(X^{\prime}\right) P_{i}(X)+S_{i}(X) h_{i}(X),
$$

where $c_{i}\left(X^{\prime}\right), S_{i}(X)$ are smooth and homogeneous. Hence we have

$$
\begin{aligned}
A(D) & =\left\{h_{i}(D)+c_{i}\left(D^{\prime}\right)\right\}\left\{P_{i}(D)+S_{i}(D)\right\}+\{\{m-2\}\} \\
& =\tilde{h}_{i}(D) \widetilde{P}_{i}(D)+\{\{m-2\}\}
\end{aligned}
$$

where

$$
\begin{aligned}
& \tilde{h}_{i}(D)=D_{t}-\tilde{\alpha}_{i}\left(D^{\prime}\right), \quad \tilde{\alpha}_{i}\left(D^{\prime}\right)=\alpha_{i}\left(D^{\prime}\right)-c_{i}\left(D^{\prime}\right) \\
& \widetilde{P}_{i}(D)=P_{i}(D)+S_{i}(D)
\end{aligned}
$$




\section{§ 2. $\widetilde{H}(\boldsymbol{D})$ and Green's Formulas}

Let

$$
\tilde{H}(D)=\left(D_{t}-\tilde{\alpha}\left(D^{\prime}\right)\right)^{2}-\tilde{\beta}\left(D^{\prime}\right)=\tilde{h}(D)^{2}-\tilde{\beta}\left(D^{\prime}\right)
$$

be one of $\left\{\widetilde{H}_{i}(D)\right\}_{i=1, \ldots, d}$ in $\S 1$. At first, we consider

$$
\tilde{\beta}\left(X^{\prime}\right)=\beta\left(X^{\prime}\right)+\beta^{1}\left(X^{\prime}\right),
$$

where $\beta\left(X^{\prime}\right) \geq 0$ is homogeneous of degree 2 and $\beta^{1}\left(X^{\prime}\right)$ is homogeneous of degree 1. In the following, we present a sufficient condition for $\tilde{\beta}\left(D^{\prime}\right)$ to be a positive operator:

$$
\operatorname{Re}\left(\tilde{\beta}\left(D^{\prime}\right) u, u\right)_{L^{2}\left(\boldsymbol{R}^{n}\right)} \geq-C\|u\|_{L^{2}\left(\boldsymbol{R}^{n}\right)}^{2} .
$$

Lemma 2. 1. Let us assume

(i) $\beta\left(X^{\prime}\right)=\sum_{j=1}^{N} b_{j}\left(X^{\prime}\right)^{2}$

where $b_{j}\left(X^{\prime}\right)$ are smooth real-valued functions,

(ii) $\operatorname{Re} \beta^{1}\left(X^{\prime}\right) \equiv 0 \bmod \boldsymbol{b}\left(X^{\prime}\right)$,

that is,

$$
\operatorname{Re} \beta^{1}\left(X^{\prime}\right)=\sum_{k=1}^{N} c_{k}\left(X^{\prime}\right) b_{k}\left(X^{\prime}\right)
$$

where $c_{k}\left(X^{\prime}\right)$ are smooth.

Then there exist $c>0$ and $C>0$ such that

$$
\operatorname{Re}\left(\tilde{\beta}\left(D^{\prime}\right) u, u\right)_{L^{2}\left(R^{n}\right)} \geq c \sum_{j=1}^{N}\left\|b_{j}\left(D^{\prime}\right) u\right\|_{L^{2}\left(R^{n}\right)}^{2}-C\|u\|_{L^{2}\left(R^{n}\right)}^{2} .
$$

Remark. If

$$
\left\{\partial_{X}^{\prime} b_{1}\left(X_{0}^{\prime}\right), \cdots, \partial_{X}^{\prime} b_{N}\left(X_{0}^{\prime}\right)\right\}
$$

are linearly independent, then (ii) is equivalent to one of the following:

(ii) $\quad \operatorname{Re} \beta^{1}\left(Y^{\prime}\right)=0$ on $\boldsymbol{b}\left(X^{\prime}\right)=0$,

(ii) $" \quad\left|\operatorname{Re} \beta^{1}\left(X^{\prime}\right)\right| \leq C\left|\boldsymbol{b}\left(X^{\prime}\right)\right|$. 
In fact, there exist smooth functions

$$
\boldsymbol{b}^{\prime}\left(X^{\prime}\right)=\left(b_{N+1}\left(X^{\prime}\right), \cdots, b_{2 n+1}\left(X^{\prime}\right)\right),
$$

where $\boldsymbol{b}^{\prime}\left(X_{0}^{\prime}\right)=0$ and

$$
\left\{\partial_{X}^{\prime} b_{1}\left(X_{0}^{\prime}\right), \cdots, \partial_{X}^{\prime} b_{2 n+1}\left(X_{0}^{\prime}\right)\right\}
$$

are linearly independent. Let $f\left(X^{\prime}\right)$ be a smooth function, then

$$
\begin{aligned}
f\left(X^{\prime}\right) & =f\left(X^{\prime}\left(\boldsymbol{b}, \boldsymbol{b}^{\prime}\right)\right)=f\left(X^{\prime}\left(0, \boldsymbol{b}^{\prime}\right)\right)+\sum_{j=1}^{N} c_{j}\left(\boldsymbol{b}, \boldsymbol{b}^{\prime}\right) b_{j} \\
& =\left.f\left(X^{\prime}\right)\right|_{\boldsymbol{b}\left(X^{\prime}\right)=0}+\sum_{j=1}^{N} c_{j}^{\prime}\left(X^{\prime}\right) b_{j}\left(X^{\prime}\right) .
\end{aligned}
$$

Therefore

$$
\left|f\left(X^{\prime}\right)\right| \leq C \sum_{j=1}^{N}\left|b_{j}\left(X^{\prime}\right)\right|
$$

is equivalent to

$$
f\left(X^{\prime}\right)=0 \quad \text { on } \quad \boldsymbol{b}\left(X^{\prime}\right)=0,
$$

that is,

$$
f\left(X^{\prime}\right)=\sum_{j=1}^{N} c_{j}^{\prime}\left(X^{\prime}\right) b_{j}\left(X^{\prime}\right)
$$

Proof. Since

$$
b_{j}^{*}\left(D^{\prime}\right)=b_{j}\left(D^{\prime}\right)+\left(D_{\xi} \partial_{x} b_{j}\right)\left(D^{\prime}\right)+\{\{-1\}\},
$$

we have

$$
\begin{aligned}
\beta\left(D^{\prime}\right)= & \sum_{j=1}^{N} b_{j}\left(D^{\prime}\right) \circ b_{j}\left(D^{\prime}\right) \\
= & \sum b_{j}\left(D^{\prime}\right)^{2}-\sum\left(D_{\xi} b_{j}\right)\left(D^{\prime}\right) \circ\left(\partial_{x} b_{j}\right)\left(D^{\prime}\right)+\{\{0\}\} \\
= & \sum b_{j}^{*}\left(D^{\prime}\right) b_{j}\left(D^{\prime}\right)-\sum\left(D_{\xi} \partial_{x} b_{j}\right)\left(D^{\prime}\right) \circ b_{j}\left(D^{\prime}\right) \\
& \quad-\sum\left(D_{\xi} b_{j}\right)\left(D^{\prime}\right) \circ\left(\partial_{x} b_{j}\right)\left(D^{\prime}\right)+\{\{0\}\} \\
= & \sum b_{j}^{*}\left(D^{\prime}\right) b_{j}\left(D^{\prime}\right)-\frac{1}{2}\left(D_{\xi} \partial_{x} \beta\right)\left(D^{\prime}\right)+\{\{0\}\} .
\end{aligned}
$$

Then we have from the assumption (ii)

$$
\tilde{\beta}\left(D^{\prime}\right)=\beta\left(D^{\prime}\right)+\beta^{1}\left(D^{\prime}\right)
$$




$$
\begin{array}{r}
=\sum b_{j}^{*}\left(D^{\prime}\right) b_{j}\left(D^{\prime}\right)+\left\{\beta^{1}\left(D^{\prime}\right)-\frac{1}{2}\left(D_{\xi} \partial_{x} \beta\right)\left(D^{\prime}\right)\right\}+\{\{0\}\} \\
=\sum b_{j}^{*}\left(D^{\prime}\right) b_{j}\left(D^{\prime}\right)+\sum c_{j}\left(D^{\prime}\right) b_{j}\left(D^{\prime}\right)+i d\left(D^{\prime}\right)+\{\{0\}\} . \\
\left(d\left(x^{\prime}\right) ; \text { real }\right)
\end{array}
$$

Hence we have

$$
\begin{aligned}
\operatorname{Re}\left(\tilde{\beta}\left(D^{\prime}\right) u, u\right) & \geq \sum\left\|b_{j}\left(D^{\prime}\right) u\right\|^{2}-C\left\{\sum\left\|b_{j}\left(D^{\prime}\right) u\right\| \cdot\|u\|+\|u\|^{2}\right\} \\
& \geq \frac{1}{2} \sum\left\|b_{j}\left(D^{\prime}\right) u\right\|^{2}-C^{\prime}\|u\|^{2}
\end{aligned}
$$

Next, we consider $\tilde{H}(D)$.

Lemma 2. 2 (Green's formula). Let us assume

(i) $\quad \beta\left(X^{\prime}\right)=\sum_{j=1}^{N} b_{j}\left(X^{\prime}\right)^{2}$,

(ii) $\beta^{1}\left(X^{\prime}\right)-\frac{1}{2} D_{\xi} \partial_{x} \beta\left(X^{\prime}\right) \equiv 0 \bmod \boldsymbol{b}\left(X^{\prime}\right)$,

(iii) $\quad\left\{h(X), b_{j}\left(X^{\prime}\right)\right\} \equiv 0 \quad \bmod \boldsymbol{b}\left(X^{\prime}\right)$,

where

$$
\left\{h(X), b_{j}\left(X^{\prime}\right)\right\}=\left(D_{\xi} h\right)\left(X^{\prime}\right) \partial_{x} b_{j}\left(X^{\prime}\right)-D_{\xi} b_{j}\left(X^{\prime}\right)\left(\partial_{x} h\right)\left(X^{\prime}\right) .
$$

Then re have

$$
\begin{aligned}
(\tilde{H}(D) u, \tilde{h}(D) u)-(\tilde{h}(D) u, \tilde{H}(D) u) \\
\quad=D_{t}\left\{\|\tilde{h}(D) u\|^{2}+\sum_{j=1}^{N}\left\|b_{j}\left(D^{\prime}\right) u\right\|^{2}\right\}+\cdots,
\end{aligned}
$$

where

$$
|\cdots| \leq C\left\{\|h(D) u\|^{2}+\sum_{j=1}^{N}\left\|b_{j}\left(D^{\prime}\right) u\right\|^{2}+\|u\|^{2}\right\}
$$

Proof. From the assumptions (i) and (ii), we have

$$
\begin{gathered}
(\tilde{H} u, \tilde{h} u)-(\tilde{h} u, \tilde{H} u) \\
=\left\{\left(\left(D_{t}-\tilde{\alpha}\right) \tilde{h} u, \tilde{h} u\right)-\left(\tilde{h} u,\left(D_{t}-\tilde{\alpha}\right) \tilde{h} u\right)\right\} \\
-\{(\tilde{\beta} u, \tilde{h} u)-(\tilde{h} u, \tilde{\beta} u)\}
\end{gathered}
$$




$$
=D_{t}\|\tilde{h} u\|^{2}-\sum_{j=1}^{N}\left\{\left(b_{j} u, b_{j} \tilde{h} u\right)-\left(b_{j} \tilde{h} u, b_{j} u\right)\right\}+\cdots .
$$

From the assumption (iii), we have

$$
\left\|\left(b_{j} \tilde{h}-\tilde{h} b_{j}\right) u\right\| \leq C\left\{\sum_{j=1}^{N}\left\|b_{j} u\right\|+\|u\|\right\}
$$

therefore

$$
\begin{aligned}
& \sum_{j=1}^{N}\left\{\left(b_{j} u, b_{j} \tilde{h} u\right)-\left(b_{j} \tilde{h} u, b_{j} u\right)\right\} \\
& \quad=\sum_{j=1}^{N}\left\{\left(b_{j} u,\left(D_{t}-\alpha\right) b_{j} u\right)-\left(\left(D_{t}-\alpha\right) b_{j} u, b_{j} u\right)\right\}+\cdots \\
& \quad=-D_{t} \sum_{j=1}^{N}\left\|b_{j} u\right\|^{2}+\cdots
\end{aligned}
$$

Corollary. There exists $\gamma_{0}>0$ such that for $\gamma>\gamma_{0}$

$$
\begin{aligned}
& e^{-2 r t}\left\{\|\tilde{h} u(t)\|^{2}+\sum_{j=1}^{N}\left\|b_{j} u(t)\right\|^{2}\right\} \\
& \quad+\gamma \int_{0}^{t} e^{-2 r t}\left\{\|\tilde{h} u(t)\|^{2}+\sum_{j=1}^{N}\left\|b_{j} u(t)\right\|^{2}\right\} d t \\
& \leq\left\{\|\tilde{h} u(0)\|^{2}+\sum_{j=1}^{N}\left\|b_{j} u(0)\right\|^{2}\right\} \\
& +C\left\{\frac{1}{\gamma} \int_{0}^{t} e^{-2 r t}\|\tilde{H} u(t)\|^{2} d t+\int_{0}^{t} e^{-2 r t}\|u(t)\|^{2} d t\right\} .
\end{aligned}
$$

Proof. In general, we have

$$
\begin{aligned}
\int_{0}^{t} e^{-2 \gamma t} D_{t} f(t) d t & =\int_{0}^{t}\left(D_{t}-2 i \gamma\right) e^{-2 \gamma t} f(t) d t \\
& =-i\left\{e^{-2 \gamma t} f(t)-f(0)+2 \gamma \int_{0}^{t} e^{-2 \gamma t} f(t) d t\right\} .
\end{aligned}
$$

Let us multiply the both sides of green's formula in Lemma 2.2 by $i e^{-2 r t}$ and integrate them, then we have

$$
\begin{aligned}
& -2 \int_{0}^{t} e^{-2 \gamma t} \operatorname{Im}(\tilde{H} u(t), \tilde{h} u(t)) d t \\
& \quad=e^{-2 \gamma t}\left\{\|\tilde{h} u(t)\|^{2}+\sum_{j=1}^{N}\left\|b_{j} u(t)\right\|^{2}\right\}-\left\{\|\tilde{h} u(0)\|^{2}+\sum_{j=1}^{N}\left\|b_{j} u(0)\right\|^{2}\right\}
\end{aligned}
$$




$$
+2 \gamma \int_{0}^{t} e^{-2 \gamma t}\left\{\|\tilde{h} u(t)\|^{2}+\sum_{j=1}^{N}\left\|b_{j} u(t)\right\|^{2}\right\} d t+\cdots,
$$

where

$$
|\cdots| \leq C \int_{0}^{t} e^{-2 \gamma t}\left\{\|\tilde{h} u(t)\|^{2}+\sum_{j=1}^{N}\left\|b_{j} u(t)\right\|^{2}+\|u(t)\|^{2}\right\} d t \text {. (Q.E.D.) }
$$

Finally, we consider of

$$
\tilde{h}(D)=D_{t}-\widetilde{\alpha}\left(D^{\prime}\right), \quad \widetilde{\alpha}\left(D^{\prime}\right)=\alpha\left(D^{\prime}\right)+\alpha^{1}\left(D^{\prime}\right),
$$

where $\alpha\left(X^{\prime}\right)$ is real-valued and homogeneous of degree 1 and $\alpha^{1}\left(X^{\prime}\right)$ is homogeneous of degree 0 . Then we have

$$
\begin{aligned}
(\tilde{h} u, u)-(u, \tilde{h} u) & =\left(\left(D_{t}-\tilde{\alpha}\right) u, u\right)-\left(u,\left(D_{t}-\tilde{\alpha}\right) u\right) \\
& =D_{t}\|u\|^{2}+\cdots,
\end{aligned}
$$

where

$$
|\cdots| \leq C\|u\|^{2}
$$

Hence we have

Lemma 2. 3. There exists $\gamma_{0}>0$ such that for $\hat{\imath}>\gamma_{0}$

$$
\begin{aligned}
e^{-2 \gamma t}\|u(t)\|^{2}+\gamma \int_{0}^{t} e^{-2 \gamma t}\|u(t)\|^{2} d t \\
\leq\|u(0)\|^{2}+\frac{C}{\gamma} \int_{0}^{t} e^{-2 \gamma t}\|\tilde{h} u(t)\|^{2} d t
\end{aligned}
$$

Here we have from the corollary of Lemma 2.2 and Lemma 2.3

Proposition 2. 4. Let us assume the conditions (i) $\sim$ (iii) stated in Lemma 2.2, then there exists $\gamma_{0}>0$ such that for $r>\gamma_{0}$

$$
\begin{aligned}
& e^{-2 \gamma t}\left\{\|h u(t)\|^{2}+\sum_{j=1}^{N}\left\|b_{j} u(t)\right\|^{2}+\gamma^{2}\|u(t)\|^{2}\right\} \\
& \quad+\gamma \int_{0}^{t} e^{-2 \tau t}\left\{\|h u(t)\|^{2}+\sum_{j=1}^{N}\left\|b_{j} u(t)\right\|^{2}+\gamma^{2}\|u(t)\|^{2}\right\} d t \\
& \leq C\left\{\|h u(0)\|^{2}+\sum_{j=1}^{N}\left\|b_{j} u(0)\right\|^{2}+\gamma^{2}\|u(0)\|^{2}\right.
\end{aligned}
$$




$$
\left.+\frac{1}{\gamma} \int_{0}^{t} \mathcal{c}^{-2 \gamma t}\|\widetilde{H} u(t)\|^{2} d t\right\} .
$$

\section{§. Condition (B)}

Let the decomposition of $A_{0}(X)$ at $X^{\prime \prime}=X_{0}^{\prime}$ be

$$
A_{0}(X)=C \prod_{i=1}^{d} H_{i}(X) \prod_{i=d+1}^{m-d} h_{i}(X)
$$

where

$$
\begin{gathered}
H_{i}(X)=h_{i}(X)^{2}-\beta_{i}\left(X^{\prime}\right)=\left(\tau-\alpha_{i}\left(X^{\prime}\right)\right)^{2}-\beta_{i}\left(X^{\prime}\right) \quad(i=1, \cdots, d), \\
h_{i}(X)=\tau-\alpha_{i}\left(X^{\prime}\right) \quad(i=d+1, \cdots, m-d) .
\end{gathered}
$$

Condition (B). It holds in a neighlorhood of $\mathrm{X}_{0}^{\prime}$ for $i=1, \cdots, d$

$$
\begin{aligned}
& \beta_{i}\left(X^{\prime}\right)=\sum_{j=1}^{N_{i}} b_{j}^{(i)}\left(X^{\prime}\right)^{2}, \\
& L_{A}\left(\alpha_{i}\left(X^{\prime}\right), X^{\prime}\right) \equiv 0 \quad \bmod b^{(i)}\left(X^{\prime}\right),
\end{aligned}
$$

where

$$
L_{A}(X)=A_{1}(X)-\frac{1}{2}\left(D_{\xi} \partial_{x} A_{0}\right)\left(X^{x}\right)
$$

(B. 3) $\quad\left\{h_{i}(X), b_{j}^{(i)}\left(X^{\prime}\right)\right\} \equiv 0 \quad \bmod b^{(i)}\left(X^{\prime}\right) \quad\left(j=1, \cdots, N_{i}\right)$,

(B. 4) $\quad\left\{b_{j}^{(i)}\left(X^{\prime}\right), b_{k}^{(i)}\left(X^{\prime}\right)\right\} \equiv 0 \quad \bmod b^{(i)}\left(X^{\prime}\right) \quad\left(j, k=1, \cdots, N_{i}\right)$.

Lemma 3. 1. (B. 2) is equivalent to

$$
L_{\beta_{i}}\left(X^{\prime}\right)=\beta_{i}^{1}\left(X^{\prime}\right)-\frac{1}{2} D_{\xi} \partial_{x} \beta_{i}\left(X^{\prime}\right) \equiv 0 \quad \bmod b^{(i)}\left(X^{\prime}\right) .
$$

Proof. Since

$$
\begin{aligned}
A(D)=A_{0}(D)+A_{1}(D) & +\{\{m-2\}\}=\widetilde{H}_{i}(D) \widetilde{P}_{i}^{\prime}(D)+\{\{m-2\}\} \\
=H_{i}(D) \circ P_{i}^{\prime}(D) & +\left\{H_{i}^{1}(D) \circ P_{i}^{\prime}(D)+H_{i}(D) \circ P_{i}^{\prime 1}(D)\right. \\
& \left.+D_{\xi} H_{i}(D) \circ \partial_{x} P_{i}^{\prime}(D)\right\}+\{\{m-2\}\},
\end{aligned}
$$

where 


$$
\begin{aligned}
\tilde{H}_{i}(D)= & H_{i}(D)+H_{i}^{1}(D)+\{\{0\}\} \\
= & H_{i}(D)+\left\{-2 h_{i}(D) \circ \alpha_{i}^{1}\left(D^{\prime}\right)+\left(D_{\xi} h_{i}\right)\left(D^{\prime}\right)\left(\partial_{x} h_{i}\right)\left(D^{\prime}\right)\right. \\
& \left.\quad-\beta_{i}^{1}\left(D^{\prime}\right)\right\}+\{\{0\}\}, \\
\widetilde{P}_{i}^{\prime}(D)= & P_{i}^{\prime}(D)+P_{i}^{\prime 1}(D)+\{\{m-4\}\},
\end{aligned}
$$

we have

$$
\left\{\begin{array}{l}
A_{0}(X)=H_{i}(X) P_{i}^{\prime}(X) \\
A_{1}(X)=H_{i}^{1}(X) P_{i}^{\prime}(X)+H_{i}(X) P_{i}^{\prime 1}(X)+D_{\xi} H_{i}(X) \partial_{x} P_{i}^{\prime}(X)
\end{array}\right.
$$

Hence we have

$$
\begin{aligned}
L_{A}(X)= & A_{1}(X)-\frac{1}{2} D_{\xi} \partial_{x} A_{0}(X) \\
= & H_{i}^{1}(X) P_{i}^{\prime}(X)+H_{i}(X) P_{i}^{\prime 1}(X)+D_{\xi} H_{i}(x) \partial_{x} P_{i}^{\prime}(X) \\
& -\frac{1}{2}\left\{\left(D_{\xi} \partial_{x} H_{i}\right)(X) P_{i}^{\prime}(X)+\left(D_{\xi} H_{i}\right)(X)\left(\partial_{x} P_{i}^{\prime}\right)(X)\right. \\
& \left.+\left(\partial_{x} H_{i}\right)(X)\left(D_{\xi} P_{1}^{\prime}\right)(X)+H_{i}(X)\left(D_{\xi} \partial_{x} P_{i}^{\prime}\right)(X)\right\},
\end{aligned}
$$

therefore

$$
\begin{aligned}
& L_{A}\left(\alpha_{i}\left(X^{\prime}\right), X^{\prime}\right) \equiv\left\{H_{i}^{1}\left(\alpha_{i}\left(X^{\prime}\right), X^{\prime}\right)\right. \\
&\left.-\frac{1}{2} D_{\xi} \partial_{x} H_{i}\left(\alpha_{i}\left(x^{\prime}\right), X^{\prime}\right)\right\} P_{1}^{\prime}\left(\alpha_{i}\left(X^{\prime}\right), X^{\prime}\right) \\
&=-\left\{\beta_{i}^{1}\left(X^{\prime}\right)-\frac{1}{2} D_{\xi} \partial_{x} \beta_{i}\left(X^{\prime}\right)\right\} P_{i}^{\prime}\left(\alpha_{i}\left(X^{\prime}\right), X^{\prime}\right) \\
& \bmod \boldsymbol{b}^{(i)}\left(X^{\prime}\right) .
\end{aligned}
$$$$
\text { Q.E.D. }
$$

Now, for examples, let us consider $A$ with constant coefficients. Of course, (B. 3) and (B. 4) are trivially satisfied. When (B. 1) is satisfied, (B. 2) is stated as

$$
A_{1}\left(\alpha_{i}\left(\xi^{\prime}\right), \xi^{\prime}\right) \equiv 0 \bmod \boldsymbol{b}^{(i)}\left(\xi^{\prime}\right),
$$

that is,

$$
\beta_{i}^{1}\left(\xi^{\prime}\right) \equiv 0 \quad \bmod \boldsymbol{b}^{(i)}\left(\xi^{\prime}\right)
$$


We shall see that $A\left(\tau, \xi^{\prime}\right)$ is hyperbolic with respect to $\tau$ if and only if

$$
\left|\beta_{i}^{1}\left(\xi^{\prime}\right)\right| \leq C \sqrt{\beta_{i}\left(\xi^{\prime}\right)} \quad(i=1, \cdots, d)
$$

In fact, we know that $A\left(\tau, \xi^{\prime}\right)$ is hyperbolic with respect to $\tau$, if and only if $([4])$

(*) $\quad\left|A_{1}\left(\tau, \xi^{\prime}\right)\right| \leq \frac{C}{|\operatorname{Im} \tau|}\left|A_{0}\left(\tau, \hat{\xi}^{\prime}\right)\right|$ for $\operatorname{Im} \tau \neq 0, \xi^{\prime} \in \mathbb{R}^{n}$.

Let us denote

$$
A_{1}\left(\tau, \xi^{\prime}\right)=\sum_{j=1}^{m-d} c_{j}\left(\xi^{\prime}\right) P_{j}\left(\tau, \xi^{\prime}\right)+\sum_{j=1}^{d} d_{j}\left(\xi^{\prime}\right) P_{j}^{\prime}\left(\tau, \xi^{\prime}\right) \quad\left(d_{j}=-\beta_{j}^{1}\right) .
$$

Since

$$
\begin{aligned}
H_{i}\left(\tau, \xi^{\prime}\right)=\{ & \left.\left(\operatorname{Re} \tau-\alpha_{i}\left(\xi^{\prime}\right)\right)^{2}-\beta_{i}\left(\xi^{\prime}\right)-(\operatorname{Im} \tau)^{2}\right\} \\
& +2 i \operatorname{Im} \tau\left(\operatorname{Re} \tau-\alpha_{i}\left(\xi^{\prime}\right)\right),
\end{aligned}
$$

we have

$$
\left|H_{i}\left(\tau, \xi^{\prime}\right)\right| \geq c|\operatorname{Im} \tau|\left\{\sqrt{\beta_{i}\left(\xi^{\prime}\right)}+|\operatorname{Im} \tau|\right\} \quad(c>0) .
$$

Therefore $(*)$ is equivalent to

$$
\left|d_{i}\left(\xi^{\prime}\right)\right| \leq C\left(\sqrt{\beta_{i}\left(\xi^{\prime}\right)}+|\operatorname{Im} \tau|\right),
$$

that is,

$$
\left|d_{i}\left(\xi^{\prime}\right)\right| \leq C \sqrt{\beta_{i}\left(\xi^{\prime}\right)}
$$

Hence we may say that (B. 2) is an almost necessary condition for $A$ to be hyperbolic in constant coefficient case. In fact, if

$$
\left\{\partial_{\xi}^{\prime} b_{i}^{(i)}\left(\xi_{0}^{\prime}\right), \cdots, \partial_{\xi}^{\prime} b_{N_{i}}^{(i)}\left(\xi_{0}^{\prime}\right)\right\}
$$

are linearly independent, (B. 2) is really necessary for $A$ to be hyperbolic.

Lemma 3.2. Let

$$
\begin{aligned}
& \beta_{i}\left(X^{\prime}\right)=\sum_{j=1}^{N_{i}} b_{j}^{(i)}\left(X^{\prime}\right)^{2}, \\
& b_{j}^{(i)}\left(X^{\prime}\right)=\sum_{k=1}^{N_{i}} c_{j k}^{(i)}\left(X^{\prime}\right) B_{k}^{(i)}\left(X^{\prime}\right), \operatorname{det}\left(c_{j k}^{(i)}\left(X^{\prime}\right)\right)_{j, k=1, \ldots, N_{i}} \neq 0,
\end{aligned}
$$


then (B. 2), (B. 3), (B. 4) are equivalent to

(B. 2)' $L_{A}\left(\alpha_{i}\left(X^{\prime}\right), X^{\prime}\right) \equiv 0 \bmod \boldsymbol{B}^{(i)}\left(X^{\prime}\right)$,

$\left(\right.$ B. 3) ${ }^{\prime} \quad\left\{h_{i}(X), B_{j}^{(i)}\left(X^{\prime}\right)\right\} \equiv 0 \quad \bmod \boldsymbol{B}^{(i)}\left(X^{\prime}\right) \quad\left(j=1, \cdots, N_{i}\right)$,

(B. 4) $\quad\left\{B_{j}^{(i)}\left(X^{\prime}\right), B_{k}^{(i)}\left(X^{\prime}\right)\right\} \equiv 0 \quad \bmod \mathbb{B}^{(i)}\left(X^{\prime}\right) \quad\left(j, k=1, \cdots, N_{i}\right)$,

respectively.

Proof. (i) Let

$$
f\left(X^{\prime}\right)=\sum_{j=1}^{N_{i}} \varphi_{j}\left(X^{\prime}\right) b_{j}^{(i)}\left(X^{\prime}\right),
$$

then

$$
f\left(X^{\prime}\right)=\sum_{k=1}^{N_{i}}\left(\sum_{j=1}^{N_{i}} \varphi_{j}\left(X^{\prime}\right) c_{j k}^{(i)}\left(X^{\prime}\right)\right) B_{k}^{(i)}\left(X^{\prime}\right)
$$

Hence,

$$
f\left(X^{\prime}\right) \equiv 0 \bmod \boldsymbol{b}^{(i)}\left(X^{\prime}\right)
$$

is equivalent to

$$
f\left(X^{\prime}\right) \equiv 0 \bmod \boldsymbol{B}^{(i)}\left(X^{\prime}\right)
$$

$$
\begin{aligned}
\left\{h_{i}(X), b_{j}^{(i)}\left(X^{\prime}\right)\right\}=\left\{h_{i}(X), \sum_{k=1}^{N_{i}} c_{j k}^{(i)}\left(X^{\prime}\right) B_{k}^{(i)}\left(X^{\prime}\right)\right\} \\
\equiv \sum_{k=1}^{N_{i}} c_{j k}^{(i)}\left(X^{\prime}\right)\left\{h_{i}(X), B_{k}^{(i)}\left(X^{\prime}\right)\right\} \bmod \boldsymbol{B}^{(i)}\left(X^{\prime}\right) .
\end{aligned}
$$

(iii) $\quad\left\{b_{j}^{(i)}\left(X^{\prime}\right), b_{k}^{(i)}\left(X^{\prime}\right)\right\} \equiv \sum_{p, q=1}^{N_{i}} c_{j p}^{(i)}\left(X^{\prime}\right) c_{k q}^{(i)}\left(X^{\prime}\right)\left\{B_{p}^{(i)}\left(X^{\prime}\right), B_{q}^{(i)}\left(X^{\prime}\right)\right\}$

$$
\bmod \boldsymbol{B}^{(i)}\left(X^{\prime}\right)
$$

Lemma 3. 3. Let us consider a special case when

$$
\beta_{i}\left(X^{\prime}\right)=\left|\boldsymbol{c}\left(x^{\prime}\right)\right|^{2}\left|\boldsymbol{B}\left(\xi^{\prime}\right)\right|^{2}=\sum_{j=1}^{M} c_{j}\left(x^{\prime}\right)^{2} \sum_{k=1}^{M} B_{k}\left(\xi^{\prime}\right)^{2}
$$

and

$$
\alpha_{i}\left(X^{\prime}\right) \equiv 0 \bmod \boldsymbol{b}\left(X^{\prime}\right)=\left(c_{j}\left(x^{\prime}\right) B_{k}\left(\xi^{\prime}\right)\right)_{j, k=1, \ldots, M} .
$$

Then, (B. 3) and (B.4) are satisfied. Moreover (B. 2) ${ }_{i}$ is equivalent 
to

$$
A_{1}\left(0, X^{\prime}\right) \equiv 0 \bmod b\left(X^{\prime}\right)
$$

Proof. Since

$$
\alpha_{i}=\sum_{j, k=1}^{M} \varphi_{j k}\left(X^{\prime}\right) c_{j}\left(x^{\prime}\right) B_{k}\left(\xi^{\prime}\right),
$$

we have

$$
\begin{aligned}
& \partial_{x}^{\prime} \alpha_{i}=\sum_{j, k} \partial_{x}^{\prime}\left\{\varphi_{j k}\left(X^{\prime}\right) c_{j}\left(x^{\prime}\right)\right\} B_{k}\left(\xi^{\prime}\right) \equiv 0 \bmod \mathbb{B}\left(\xi^{\prime}\right), \\
& \partial_{\xi}^{\prime} \alpha_{i}=\sum_{j, k} \partial_{\xi}^{\prime}\left\{\varphi_{j k}\left(X^{\prime}\right) B_{k}\left(\xi^{\prime}\right)\right\} c_{j}\left(x^{\prime}\right) \equiv 0 \bmod \boldsymbol{c}\left(x^{\prime}\right), \\
& \partial_{t} \alpha_{i}=\sum_{j, k} \partial_{t} \varphi_{j k}\left(X^{\prime}\right) c_{j}\left(x^{\prime}\right) B_{k}\left(\xi^{\prime}\right) \equiv 0 \bmod \mathbb{b}\left(x^{\prime}, \xi^{\prime}\right) .
\end{aligned}
$$

Hence

$$
\begin{aligned}
\left\{h_{i}, c_{j} B_{k}\right\} & =-\left\{\alpha_{i}, c_{j} B_{k}\right\} \\
= & -\left(D_{\xi}^{\prime} \alpha_{i}\right)\left(\partial_{x}^{\prime} c_{j}\right) B_{k}+\left(\partial_{x}^{\prime} \alpha_{i}\right) c_{j}\left(D_{\xi}^{\prime} B_{k}\right) \equiv 0 \bmod b, \\
\left\{c_{j} B_{k}, c_{p} B_{q}\right\} & =c_{j}\left(D_{\xi}^{\prime} B_{k}\right)\left(\partial_{x}^{\prime} c_{p}\right) B_{q}-\left(\partial_{x}^{\prime} c_{j}\right) B_{k} c_{p}\left(D_{\xi}^{\prime} B_{q}\right) \equiv 0 \bmod b,
\end{aligned}
$$

and

$$
\begin{aligned}
L_{A}\left(\alpha_{i}\left(X^{\prime}\right), X^{\prime}\right) \equiv L_{A}\left(0, X^{\prime}\right) \\
\quad=A_{1}\left(0, X^{\prime}\right)-\frac{1}{2}\left(D_{\xi} \partial_{x} H_{i}\right)\left(0, X^{\prime}\right) P_{i}^{\prime}\left(0, X^{\prime}\right),
\end{aligned}
$$

where

$$
\begin{array}{rr}
\frac{1}{2}\left(D_{\xi} \partial_{x} H_{i}\right)\left(0, X^{\prime}\right) \equiv\left(D_{\xi} h_{i}\right)\left(\partial_{x} h_{i}\right)=-\frac{1}{i} \partial_{t} \alpha_{i}+\left(D_{\xi}^{\prime} \alpha_{i}\right)\left(\partial_{x}^{\prime} \alpha_{i}\right) \equiv 0 \\
\bmod b & \text { Q.E.D. }
\end{array}
$$

Example 1. Let

$$
A_{0}=\tau^{2}-2 c\left(x^{\prime}\right)\left(\xi_{1}+\xi_{2}\right) \tau+2 c\left(x^{\prime}\right)^{2} \xi_{1} \xi_{2},
$$

that is,

$$
A_{0}=(\tau-\alpha)^{2}-\beta, \quad \alpha=c\left(x^{\prime}\right)\left(\xi_{1}+\xi_{2}\right), \quad \beta=c\left(x^{\prime}\right)^{2}\left(\xi_{1}^{2}+\xi_{2}^{2}\right) .
$$

Then, (B. 3) and (B. 4) are satisfied, owing to Lemma 3.3. Let 


$$
A_{1}=a_{0}\left(t, x^{\prime}\right) \tau+a_{1}\left(t, x^{\prime}, \xi^{\prime}\right),
$$

then (B. 2) is equivalent to

$$
a_{1}\left(t, x^{\prime}, \xi^{\prime}\right)=\varphi\left(t, x^{\prime}\right) c\left(x^{\prime}\right) \xi_{1}+\psi\left(t, x^{\prime}\right) c\left(x^{\prime}\right) \xi_{2} \text { 。 }
$$

Example 2. Let

$$
\begin{aligned}
& A_{0}=\tau^{4}-2\left|\xi^{\prime}\right|^{2} \tau^{2}+c\left(x^{\prime}\right)^{2}\left(\xi_{1}{ }^{4}+\xi_{2}{ }^{4}\right) \quad\left(0 \leq c\left(x^{\prime}\right)<1\right) \\
&=\left\{\tau^{2}-\left(\left|\xi^{\prime}\right|^{2}+\left[\left|\xi^{\prime}\right|^{4}-c\left(x^{\prime}\right)^{2}\left(\xi_{1}{ }^{4}+\xi_{2}{ }^{4}\right)\right]^{1 / 2}\right)\right\} \\
& \times\left\{\tau^{2}-\frac{c\left(x^{\prime}\right)^{2}\left(\xi_{1}^{4}+\xi_{2}^{4}\right)}{\left|\xi^{\prime}\right|^{2}+\left[\left|\xi^{\prime}\right|^{4}-c\left(x^{\prime}\right)^{2}\left(\xi_{1}^{4}+\xi_{2}{ }^{4}\right)\right]^{1 / 2}}\right\} .
\end{aligned}
$$

Then, $A_{0}=0$ has a unique double root $\tau=0$ at $c\left(x^{\prime}\right)^{2}\left(\xi_{1}^{4}+\xi_{2}^{4}\right)=0$. (B. 3) and (B. 4) are satisfied, owing to Lemma 3. 3. Let

$$
A_{1}=a_{0}\left(t, x^{\prime}\right) \tau^{3}+a_{1}\left(t, x^{\prime}, \xi^{\prime}\right) \tau^{2}+a_{2}\left(t, x^{\prime}, \xi^{\prime}\right) \tau+a_{3}\left(t, x^{\prime}, \xi^{\prime}\right),
$$

then (B. 2) is equivalent to

$$
a_{3}\left(t, x^{\prime}, \xi^{\prime}\right)=\sum_{j=1}^{n} \varphi_{j}\left(t, x^{\prime}\right) \xi_{j} c\left(x^{\prime}\right) \xi_{1}^{2}+\sum_{j=1}^{n} \psi_{j}\left(t, x^{\prime}\right) \xi_{j} c\left(x^{\prime}\right) \xi_{2}^{2} 。
$$

Example 3. Let the characteristic roots of $A_{0}$ be smooth and $d=1$ at most. Let

$$
A_{0}(X)=\left(\tau-\lambda\left(X^{\prime}\right)\right)\left(\tau-\mu\left(X^{\prime}\right)\right) P^{\prime}(X)=H(X) P^{\prime}(X),
$$

where $\lambda\left(X_{0}^{\prime}\right)=\mu\left(X_{0}^{\prime}\right)$, then

$$
H(X)=\left(\tau-\alpha\left(X^{\prime}\right)\right)^{2}-\beta\left(X^{\prime}\right),
$$

where

$$
\alpha\left(X^{\prime}\right)=\frac{\lambda\left(X^{\prime}\right)+\mu\left(X^{\prime}\right)}{2}, \beta\left(X^{\prime}\right)=\left(\frac{\lambda\left(X^{\prime}\right)-\mu\left(X^{\prime}\right)}{2}\right)^{2} .
$$

Hence, (B. 1) is satisfied by setting

$$
b\left(X^{\prime}\right)=\lambda\left(X^{\prime}\right)-\mu\left(X^{\prime}\right) .
$$

Then, (B. 2) means

$$
A_{1}\left(\lambda\left(X^{\prime}\right), X^{\prime}\right)-\frac{1}{2}\left\{D_{\xi}\left(\tau-\lambda\left(X^{\prime}\right)\right) \partial_{x}\left(\tau-\mu\left(X^{\prime}\right)\right)\right.
$$




$$
\begin{aligned}
& \left.+D_{\xi}\left(\tau-\mu\left(X^{\prime}\right)\right) \partial_{x}\left(\tau-\lambda\left(X^{\prime}\right)\right)\right\} \\
& \times \frac{1}{2} \partial_{\tau}^{2} A_{0}\left(\lambda\left(X^{\prime}\right), X^{\prime}\right) \equiv 0 \bmod \lambda\left(X^{\prime}\right)-\mu\left(X^{\prime}\right)
\end{aligned}
$$

that is,

$$
\begin{aligned}
A_{1}\left(\lambda\left(X^{\prime}\right), X^{\prime}\right) & -\frac{1}{2 i}\left\{-\partial_{t} \lambda\left(X^{\prime}\right)-\partial_{t} \mu\left(X^{\prime}\right)\right. \\
& \left.+\partial_{\xi}^{\prime} \lambda\left(X^{\prime}\right) \partial_{x}^{\prime} \mu\left(X^{\prime}\right)+\partial_{\xi}^{\prime} \mu\left(X^{\prime}\right) \partial_{x}^{\prime} \lambda\left(X^{\prime}\right)\right\} \\
& \times \frac{1}{2} \partial_{\tau}^{2} A_{0}\left(\lambda\left(X^{\prime}\right), X^{\prime}\right) \equiv 0 \bmod \lambda\left(X^{\prime}\right)-\mu\left(X^{\prime}\right)
\end{aligned}
$$

(B. 3) means

$$
\begin{aligned}
& \left\{\tau-\frac{\lambda\left(X^{\prime}\right)+\mu\left(X^{\prime}\right)}{2}, \lambda\left(X^{\prime}\right)-\mu\left(X^{\prime}\right)\right\} \\
& \quad=\left\{\tau-\lambda\left(X^{\prime}\right), \tau-\mu\left(X^{\prime}\right)\right\} \equiv 0 \bmod \lambda\left(X^{\prime}\right)-\mu\left(X^{\prime}\right),
\end{aligned}
$$

that is,

$$
\begin{gathered}
D_{\xi}\left(\tau-\lambda\left(X^{\prime}\right)\right) \partial_{x}\left(\tau-\mu\left(X^{\prime}\right)\right)-D_{\xi}\left(\tau-\mu\left(X^{\prime}\right)\right) \partial_{x}\left(\tau-\lambda\left(X^{\prime}\right)\right) \equiv 0 \\
\bmod \lambda\left(X^{\prime}\right)-\mu\left(X^{\prime}\right) .
\end{gathered}
$$

Moreover, (B. 2) and (B. 3) are equivalent to

$$
\left\{\begin{aligned}
A_{1}\left(\lambda\left(X^{\prime}\right), X^{\prime}\right) & -D_{\xi}\left(\tau-\lambda\left(X^{\prime}\right)\right) \partial_{x}\left(\tau-\mu\left(X^{\prime}\right)\right) \\
\times & \frac{1}{2} \partial_{\tau}^{2} A_{0}\left(\lambda\left(X^{\prime}\right), X^{\prime}\right) \equiv 0 \bmod \lambda\left(X^{\prime}\right)-\mu\left(X^{\prime}\right), \\
A_{1}\left(\lambda\left(X^{\prime}\right), X^{\prime}\right) & -D_{\xi}\left(\tau-\mu\left(X^{\prime}\right)\right) \partial_{x}\left(\tau-\lambda\left(X^{\prime}\right)\right) \\
& \times \frac{1}{2} \partial_{\tau}^{2} A_{0}\left(\lambda\left(X^{\prime}\right), X^{\prime}\right) \equiv 0 \bmod \lambda\left(X^{\prime}\right)-\mu\left(X^{\prime}\right) .
\end{aligned}\right.
$$

\section{§ 4. Energy Inequalities}

Already, we have had in Section 1

$$
A(D)= \begin{cases}\widetilde{H}_{i}(D) \widetilde{P}_{i}^{\prime}(D)+\{\{m-2\}\} & (i=1, \cdots, d) \\ \tilde{h}_{i}(D) \widetilde{P}_{i}(D)+\{\{m-2\}\} & (i=d+1, \cdots, m-d)\end{cases}
$$

by the simplification of $U=\mathbb{R}^{n+1} \times\left(\mathbb{R}^{n}-\{0\}\right)$. Now, we assume 
conditions (B. 1$) \sim($ B. 3$)$, then we can apply results in Section 2 .

Lemma 4. 1. There exist $\gamma_{0}>0$ and $C>0$ such that for $\gamma>\gamma_{0}$

$$
\begin{gathered}
e^{-2 \gamma t}\|\boldsymbol{P} u(t)\|^{2}+\gamma \int_{0}^{t} e^{-2 \gamma t}\|\boldsymbol{P} u(t)\|^{2} d t \\
\leq C\left\{\|\boldsymbol{P} u(0)\|^{2}+\|u(0)\|_{m-2}^{2}+\frac{1}{\gamma} \int_{0}^{t} e^{-2 \gamma t}\|A u(t)\|^{2} d t\right. \\
\left.+e^{-2 \gamma t}\|u(t)\|_{m-2}^{2}+\gamma \int_{0}^{t} e^{-2 \gamma t}\|u(t)\|_{m-2}^{2} d t\right\}
\end{gathered}
$$

where

$$
\begin{aligned}
& \|u(t)\|_{k}^{2}=\sum_{j=0}^{k}\left\|D_{t}^{j} u(t)\right\|_{H^{k-j}\left(R_{x}^{n}\right)}^{2} \\
& \|\boldsymbol{P} u(t)\|^{2}=\sum_{i=1}^{m-d}\left\|P_{i} u(t)\right\|^{2}+\sum_{i=1}^{d} \sum_{j=1}^{N_{i}}\left\|b_{j}^{(i)} P_{i}^{\prime} u(t)\right\|^{2} .
\end{aligned}
$$

Remark. Let $Q(X)$ be homogeneous of degree $m-1$ and

$$
Q\left(\alpha_{i}\left(X^{\prime}\right), X^{\prime}\right) \equiv 0 \bmod b^{(i)}\left(X^{\prime}\right) \quad(i=1, \cdots, d),
$$

then we have

$$
\|Q(D) u\| \leq C\left(\|\boldsymbol{P} u\|+\|u\|_{m-2}\right)
$$

Proof. We apply the corollary of Lemma 2.2 to

$$
A(D) u=\widetilde{H}_{i}(D) \widetilde{P}_{i}^{\prime}(D) u+\{\{m-2\}\} u \quad(i=1, \cdots, d),
$$

then we have

$$
\begin{aligned}
& e^{-2 \gamma t}\left\{\left\|\widetilde{h}_{i} \widetilde{P}_{i}^{\prime} u(t)\right\|^{2}+\sum_{j=1}^{N_{i}}\left\|b_{j}^{(i)} \widetilde{P}_{i}^{\prime} u(t)\right\|^{2}\right\} \\
& \quad+\gamma \int_{0}^{t} e^{-2 r t}\left\{\left\|\tilde{h}_{i} \widetilde{P}_{i}^{\prime} u(t)\right\|^{2}+\sum_{j=1}^{N_{i}}\left\|b_{j}^{(i)} \widetilde{P}_{i}^{\prime} u(t)\right\|^{2}\right\} d t \\
& \leq\left\{\left\|\tilde{h}_{i} \widetilde{P}_{i}^{\prime} u(0)\right\|^{2}+\sum_{j=1}^{N_{i}}\left\|b_{j}^{(i)} \widetilde{P}_{i}^{\prime} u(0)\right\|^{2}\right\} \\
& +C\left\{\frac{1}{\gamma} \int_{0}^{t} e^{-2 \gamma t}\|A u(t)\|^{2} d t+\int_{0}^{t} e^{-2 \gamma t}\|u(t)\|_{m-2}^{2} d t\right\} .
\end{aligned}
$$

On the other hand, we apply Lemma 2.3 to 


$$
A(D) u=\tilde{h}_{i}(D) \widetilde{P}_{i}(D) u+\{\{m-2\}\} u \quad(i=d+1, \cdots, m-d),
$$

then we have

$$
\begin{aligned}
& e^{-2 \gamma t}\left\|\widetilde{P}_{i} u(t)\right\|^{2}+\gamma \int_{0}^{t} e^{-2 \gamma t}\left\|\widetilde{P}_{i} u(t)\right\|^{2} d t \\
& \leq\left\|\widetilde{P}_{i} u(0)\right\|^{2}+\frac{C}{\gamma} \int_{0}^{t} e^{-2 \gamma t}\left(\|A u(t)\|^{2}+\|u(t)\|_{m-2}^{2}\right) d t .
\end{aligned}
$$

We have only to remark

$$
\left\{\begin{array}{l}
\tilde{h}_{i}(D) \widetilde{P}_{i}^{\prime}(D)=P_{i}(D)+\{\{m-2\}\} \quad(i=1, \cdots, d), \\
b_{j}^{(i)}\left(D^{\prime}\right) \widetilde{P}_{i}^{\prime}(D)=b_{j}^{(i)}\left(D^{\prime}\right) P_{i}^{\prime}(D)+\{\{m-2\}\} \quad(i=1, \cdots, d), \\
\widetilde{P}_{i}(D)=P_{i}(D)+\{\{m-2\}\} \quad(i=d+1, \cdots, m-d) .
\end{array}\right.
$$

Q.E.D.

Since $\partial_{t} A_{0}(X)$ is strictly hyperbolic, we have

\section{Lemma t. 2 .}

$$
\begin{aligned}
e^{-2 r t} \sum_{k=0}^{m-2} \gamma^{2(m-2-k)}\|u(t)\|_{k}^{2}+\gamma \int_{0}^{t} e^{-2 \gamma t} \sum_{k=0}^{m-2} \gamma^{2(m-2-k)}\|u(t)\|_{k}^{2} d t \\
\leq C\left\{\sum_{k=0}^{m-2} \gamma^{2(m-2-k)}\|u(0)\|_{k}^{2}+\frac{1}{\gamma} \int_{0}^{t} e^{-2 \tau t}\left\|\partial_{\tau} A_{0}(D) u(t)\right\|^{2} d t\right\} .
\end{aligned}
$$

Here we remark that

$$
\partial_{\tau} A_{0}(X)=2 \sum_{i=1}^{d} P_{i}(X)+\sum_{i=d+1}^{m-d} P_{i}(X),
$$

then we have

Proposition 4.3. Let us assume condition (B. 1) $\sim($ B. 3), then there exist $\gamma_{0}>0$ and $C>0$ such that for $r>\gamma_{0}$

$$
\begin{aligned}
& e^{-2 \gamma t}\left(\|\boldsymbol{P} u(t)\|^{2}\right.\left.+\sum_{k=0}^{m-2} \gamma^{2(m-1-k)}\|u(t)\| \frac{2}{k}\right) \\
&+\gamma \int_{0}^{t} e^{-2 \gamma t}\left(\|\mathbb{P} u(t)\|^{2}+\sum_{k=0}^{m-2} \gamma^{2(m-1-k)}\|u(t)\|_{k}^{2}\right) d t \\
& \leq C\left\{\left(\|\boldsymbol{P} u(0)\|^{2}+\sum_{k=0}^{m-2} \gamma^{2(m-1-k)}\|u(0)\|_{k}^{2}\right)+\frac{1}{\gamma} \int_{0}^{t} e^{-2 \gamma t}\|A u(t)\|^{2} d t\right\} .
\end{aligned}
$$


Let us denote

$$
\Lambda\left(\xi^{\prime}\right)=\left|\xi^{\prime}\right|,
$$

then we have

\section{Corollary.}

$$
\begin{aligned}
& \gamma^{2} e^{-2 \gamma t}\left\|(\Lambda+\gamma)^{-1} u(t)\right\|_{m-1}^{2}+\gamma^{3} \int_{0}^{t} e^{-2 \gamma t}\left\|(\Lambda+\gamma)^{-1} u(t)\right\|_{m-1}^{2} d t \\
& \leq C\left\{\|\boldsymbol{P} u(0)\|^{2}+\sum_{k=0}^{m-2} \gamma^{2(m-1-k)}\|u(0)\|_{k}^{2}+\frac{1}{\gamma} \int_{0}^{t} e^{-2 \gamma t}\|A u(t)\|^{2} d t\right\} .
\end{aligned}
$$

Proof. Since

$$
P_{1}(D)=D_{t}^{m-1}+a_{1}\left(D^{\prime}\right) D_{t}^{m-2}+\cdots+a_{m-1}\left(D^{\prime}\right),
$$

where $a_{i}\left(X^{\prime}\right)$ is homogeneous of degree $i$, we have

$$
\begin{aligned}
r^{2}\left\|(\Lambda+\gamma)^{-1} D_{t}^{m-1} u\right\|^{2} & \leq C \gamma^{2}\left\{\left\|(\Lambda+\gamma)^{-1} P_{1}(D) u\right\|^{2}+\|u\|_{m-2}^{2}\right\} \\
& \leq C\left(\|\boldsymbol{P} u\|^{2}+\gamma^{2}\|u\|_{m-2}^{2}\right) .
\end{aligned}
$$

Moreover, since

$$
\gamma^{2}\left\|(\Lambda+\gamma)^{-1} \Lambda^{m-1-j} D_{t}^{j} u\right\|^{2} \leq C \sum_{k=j}^{m-2} \gamma^{2(m-1-k)}\|u\|_{k}^{2} \quad(j=0,1, \cdots, m-2),
$$

we have

$$
\gamma^{2}\left\|(\Lambda+\gamma)^{-1} u\right\|_{m-1}^{2} \leq C\left(\|\boldsymbol{P} u\|^{2}+\sum_{k=0}^{m-2} \gamma^{2(m-1-k)}\|u\|_{k}^{2}\right) . \quad \text { Q.E.D. }
$$

\section{Chapter II. Energy Inequalities (continued)}

\section{§. Localizations}

Let $X_{0}^{\prime} \in \boldsymbol{R}^{n+1} \times S^{n-1}$, then there is an $r$ such that in the neighbourhood

$$
V\left(X_{0}^{\prime}\right)=\left\{X^{\prime} \in \boldsymbol{R}^{n+1} \times S^{n-1} ;\left|X^{\prime}-X_{0}^{\prime}\right|<r\right\},
$$

the decomposition of $A_{0}(X)$ holds and condition $(B)$ is satisfied. Of course, $r$ depends on $X_{0}^{\prime}$. Here we denote

$$
W\left(X_{0}^{\prime}\right)=\left\{X^{\prime} \in \boldsymbol{R}^{n+1} \times S^{n-1} ;\left|X^{\prime}-X_{0}^{\prime}\right|<\frac{r}{2}\right\} .
$$


Owing to the assumption (A), $A_{0}(X)$ is independent of $\left(t, x^{\prime}\right)$ in $\left\{t^{2}+\left|x^{\prime}\right|^{2}>R^{2}\right\}$, therefore there exist $\left\{X_{p}^{\prime}\right\}_{p=1, \cdots, M}$ such that

$$
\left\{t^{2}+\left|x^{\prime}\right|^{2} \leq R^{2}\right\} \times S^{n-1} \subset \bigcup_{p=1}^{M} W\left(X_{p}^{\prime}\right)
$$

Especially, we may think

$$
\left(t_{0}, x_{0}^{\prime}\right) \times S^{n-1} \subset \bigcup_{p=1}^{M_{0}} W\left(X_{p}^{\prime}\right) \quad\left(t_{0}^{2}+\left|x_{0}^{\prime}\right|^{2}=R^{2}, M_{0}<M\right) .
$$

Now we denote

$$
\begin{gathered}
W_{0}\left(X_{p}^{\prime}\right)=W\left(X_{p}^{\prime}\right) \cup\left\{\left(t, x^{\prime}, \xi^{\prime}\right) \in R^{n+1} \times S^{n-1} ; t^{2}+\left|x^{\prime}\right|^{2}>R^{2},\right. \\
\left.\left(t_{0}, x_{0}^{\prime}, \xi^{\prime}\right) \in W\left(X_{p}^{\prime}\right)\right\},
\end{gathered}
$$

and

$$
\left\{\begin{array}{c}
\widetilde{W}\left(X_{p}^{\prime}\right)=\left\{\left(t, x^{\prime}, \xi^{\prime}\right) \in \mathbb{R}^{n+1} \times\left(\mathbb{R}^{n}-\{0\}\right) ;\left(t, x^{\prime}, \frac{\tilde{\xi}^{\prime}}{\left|\xi^{\prime}\right|}\right) \in W_{0}\left(X_{p}^{\prime}\right)\right\} \\
\left(p=1, \cdots, M_{0}\right), \\
\widetilde{W}\left(X_{p}^{\prime}\right)=\left\{\left(t, x^{\prime}, \xi^{\prime}\right) \in \mathbb{R}^{n+1} \times\left(\mathbb{R}^{n}-\{0\}\right) ;\left(t, x^{\prime}, \frac{\xi^{\prime}}{\left|\xi^{\prime}\right|}\right) \in W\left(X_{p}^{\prime}\right)\right\} \\
\left(p=M_{0}+1, \cdots, M\right),
\end{array}\right.
$$

then we have

$$
\mathbb{R}^{n+1} \times\left(\mathbb{R}^{n}-\{0\}\right) \subset \bigcup_{p=1}^{M} \widetilde{W}\left(X_{p}^{\prime}\right)
$$

Let $W\left(X_{p}^{\prime}\right) \cap W\left(X_{q}^{\prime}\right) \neq \varnothing$, then we have $X_{p}^{\prime} \in V\left(X_{q}^{\prime}\right)$ or $X_{q}^{\prime} \in V\left(X_{p}^{\prime}\right)$. Let $X_{q}^{\prime} \in V\left(X_{p}^{\prime}\right)$, then a simple root of $A_{0}(X)=0$ at $X^{\prime}=X_{p}^{\prime}$ is always simple along the straight line from $X_{p}^{\prime}$ to $X_{q}^{\prime}$ and double roots at $X^{\prime}=X_{p}^{\prime}$ may be simple or may be double there with same pair.

Here we introduce a decomposition of unity depending on $\left\{\widetilde{W}\left(X_{p}^{\prime}\right)\right\}$, that is, there exist $\left\{\varphi_{p}\left(X^{\prime}\right)\right\}_{p=1, \cdots, M}$ such that

$$
\sum_{p=1}^{M} \varphi_{p}\left(X^{\prime}\right)=1 \quad \text { in } R^{n+1} \times\left(R^{n}-\{0\}\right), \quad \operatorname{supp}\left[\varphi_{p}\left(X^{\prime}\right)\right] \subset \widetilde{W}\left(X_{p}^{\prime}\right),
$$

where $\varphi_{p}\left(X^{\prime}\right)$ are smooth and homogeneous of degree 0 with respect to $\xi$.

Let 


$$
A_{0}(X)=\prod_{i=1}^{d_{p}} H_{p i}(X) \prod_{i=\bar{d}_{p+1}}^{m-d_{p}} h_{p i}(X)
$$

be the decomposition of $A_{0}(X)$ at $X^{\prime}=X_{p}^{\prime}$ in $\widetilde{W}\left(X_{p}^{\prime}\right)$, where

$$
\begin{aligned}
& H_{p i}(X)=h_{p i}(X)^{2}-\beta_{p i}\left(X^{\prime}\right)=\left(\tau-\alpha_{p i}\left(X^{\prime}\right)\right)^{2}-\beta_{p i}\left(X^{\prime}\right), \\
& h_{p i}(X)=\tau-\alpha_{p i}\left(X^{\prime}\right)
\end{aligned}
$$

and

$$
\beta_{p i}\left(X^{\prime}\right)=\sum_{k=1}^{N_{p i}} b_{k}^{(p i)}\left(X^{\prime}\right)^{2}
$$

Suitable extensions of $\alpha_{p i}, b_{k}^{(p i)}$ outside $\widetilde{W}\left(X_{p}^{\prime}\right)$ will be denoted by the same notations. Hence we have

$$
A_{0}^{(p)}(X)=\prod_{i=1}^{d_{p}} H_{p i}(X) \prod_{i=d_{p}+1}^{m-d_{p}} h_{p i}(X), \quad X^{\prime} \in \boldsymbol{R}^{n+1} \times\left(\boldsymbol{R}^{n}-\{0\}\right),
$$

where

$$
A_{0}^{(p)}(X)=A_{0}(X), \quad X^{\prime} \in \widetilde{W}\left(X_{p}^{\prime}\right)
$$

Now, we denote

$$
\left\{\begin{array}{l}
P_{p i}(X)=h_{p i}(X) \frac{A_{0}^{(p)}(X)}{H_{p i}(X)}, \quad P_{p i}^{\prime}(X)=\frac{A_{0}^{(p)}(X)}{H_{p i}(X)} \quad\left(i=1, \cdots, d_{p}\right), \\
P_{p i}(X)=\frac{A_{0}^{(p)}(X)}{h_{p i}(X)} \quad\left(i=d_{p}+1, \cdots, m-d_{p}\right)
\end{array}\right.
$$

and

$$
\left\|\boldsymbol{P}^{(p)}(D) u\right\|^{2}=\sum_{i=1}^{m-d_{p}}\left\|P_{p i}(D) u\right\|^{2}+\sum_{i=1}^{d_{p}} \sum_{k=1}^{N_{p i}}\left\|b_{k}^{(p i)}\left(D^{\prime}\right) P_{p i}^{\prime}(D) u\right\|^{2} .
$$

Lemma 5. 1. Let $\varphi\left(X^{\prime}\right)$ be smooth and homogeneous of degree 0 , and let its support be in $\widetilde{W}\left(X_{p}^{\prime}\right) \cap \widetilde{W}\left(X_{q}^{\prime}\right)$ then

$$
\left\|\boldsymbol{P}^{(q)}(D) \varphi\left(D^{\prime}\right) u\right\| \leq C\left\{\left\|\boldsymbol{P}^{(p)}(D) \varphi\left(D^{\prime}\right) u\right\|+\|u\|_{m-2}\right\}
$$

Proof. Let

$$
\begin{gathered}
A_{0}(X)=\prod_{i=1}^{d_{p}} H_{p i}(X) \prod_{i=d_{p+1}}^{m-d_{p}} h_{p i}(X)=\prod_{j=1}^{d_{q}} H_{q j}(X) \prod_{j=d_{q}+1}^{m-d_{q}} h_{q j}(X), \\
X^{\prime} \in \widetilde{W}\left(X_{p}^{\prime}\right) \cap \widetilde{W}\left(X_{q}^{\prime}\right)
\end{gathered}
$$


then the following cases may happen:

(i) for $j \leq d_{q}$, there exists $i \leq d_{p}$ such that $H_{q j}(X)=H_{p i}(X)$,

(ii) for $j \leq d_{q}$, there exist $i>d_{p}$ and $i^{\prime}>d_{p}$ such that $H_{q j}(X)$ $=h_{p i}(X) h_{p i^{\prime}}(X)$,

(iii) for $j>d_{q}$, there exist $j^{\prime}>d_{q}$ and $i \leq d_{p}$ such that $h_{q j}(X) h_{q j^{\prime}}(X)$ $=H_{p i}(X)$,

(iv) for $j>d_{q}$, there exists $i>d_{p}$ such that $h_{q j}(X)=h_{p i}(X)$.

Case (i) It is sufficient to show that

$$
\begin{aligned}
& \sum_{k=1}^{N_{q j}}\left\|b_{k}^{(q j)}\left(D^{\prime}\right) P_{q j}^{\prime}(D) \varphi\left(D^{\prime}\right) u\right\| \\
& \quad \leq C\left\{\sum_{k=1}^{N_{p i}}\left\|b_{k}^{(p i)}\left(D^{\prime}\right) P_{p i}^{\prime}(D) \varphi\left(D^{\prime}\right) u\right\|+\|\| u \|_{m-2}\right\}
\end{aligned}
$$

and

$$
\left\|P_{q j}(D) \varphi\left(D^{\prime}\right) u\right\| \leq C\left(\left\|P_{p i}(D) \varphi\left(D^{\prime}\right) u\right\|+\|u\|_{m-2}\right) .
$$

The latter is trivial, because

$$
\begin{aligned}
& \left\|P_{q j} \varphi u\right\| \leq\left\|P_{q j} \circ \varphi u\right\|+C\|\| u \|_{m-2} \\
& \quad=\left\|P_{p i} \circ \varphi u\right\|+C\|u\|_{m-2} \leq\left\|P_{p i} \varphi u\right\|+C^{\prime}\|u\|_{m-2} .
\end{aligned}
$$

Now, let us consider

$$
\begin{aligned}
I_{p i} & =\sum_{k=1}^{N_{p i}}\left\|b_{k}^{(p i)} P_{p i}^{\prime} \varphi u\right\|^{2} \\
& =\left(\beta_{p i} P_{p i}^{\prime} \varphi u, P_{p i}^{\prime} \varphi u\right)+\left(\left(\sum_{k=1}^{N_{p i} i} b_{k}^{(p i)^{*}} b_{k}^{(p i)}-\beta_{p i}\right) P_{p i}^{\prime} \varphi u, P_{p i}^{\prime} \varphi u\right) \\
& =K_{p i}+L_{p i} .
\end{aligned}
$$

Since

$$
\beta_{p i} P_{p i}^{\prime} \varphi-\beta_{p i} \circ P_{p i}^{\prime} \circ \varphi=D_{\xi} \beta_{p i} \circ \partial_{x} P_{p i}^{\prime} \circ \varphi+D_{\xi}\left(\beta_{p i} \circ P_{p i}^{\prime}\right) \circ \partial_{x} \varphi+\{\{m-2\}\},
$$

we have

$$
\begin{aligned}
K_{p i}=( & \left.\left(\beta_{p i} \circ P_{p i}^{\prime} \circ \varphi\right) u, P_{p i}^{\prime} \circ \varphi u\right)+\left(\left(\beta_{p i} \circ P_{p i}^{\prime} \circ \varphi\right) u, D_{\xi} P_{p i}^{\prime} \circ \partial_{x} \varphi u\right) \\
& +\left(\left\{D_{\xi} \beta_{p i} \circ \partial_{x} P_{p i}^{\prime} \circ \varphi+D_{\xi}\left(\beta_{p i} \circ P_{p i}^{\prime}\right) \circ \partial_{x} \varphi\right\} u, P_{p i}^{\prime} \circ \varphi u\right)+\cdots,
\end{aligned}
$$

where

$$
|\cdots| \leq C\|u\|_{m-2}^{2},
$$


therefore we have

$$
\left|K_{p i}-\left(\left(\beta_{p i} \circ P_{p i}^{\prime} \circ \varphi\right) u,\left(P_{p i}^{\prime} \circ \varphi\right) u\right)\right| \leq C\left(\sqrt{I_{p i}}+\|u\|_{m-2}\right)\|u\|_{m-2} .
$$

On the other hand, since

$$
\sum_{k=1}^{N_{p i}} b_{k}^{(p i)^{*}} b_{k}^{(p i)}-\beta_{p i}=\frac{1}{2} D_{\xi} \partial_{x} \beta_{p i}+\{\{0\}\} .
$$

we have

$$
L_{p i}=\frac{1}{2}\left(D_{\xi} \partial_{x} \beta_{p i} \circ P_{p i}^{\prime} \varphi u, P_{p i}^{\prime} \circ \varphi u\right)+\cdots,
$$

where

$$
\mid \cdots i \leq C\|u\|_{n-2}^{2},
$$

therefore we have

$$
\left|L_{p i}-\frac{1}{2}\left(D_{\xi} \partial_{x} \beta_{p i} \circ P_{p i}^{\prime} \circ \varphi u, P_{p i}^{\prime} \circ \varphi u\right)\right| \leq C\left(\sqrt{I_{p i}}+\|u\|_{m-2}\right)\|u\|_{m-2} .
$$

Since

$$
\left\{\begin{array}{l}
\left(\left(\beta_{p i} \circ P_{p i}^{\prime} \circ \varphi\right) u,\left(P_{p i}^{\prime} \circ \varphi\right) u\right)=\left(\left(\beta_{q j} \circ P_{q j}^{\prime} \circ \varphi\right) u,\left(P_{q j}^{\prime} \circ \varphi\right) u\right), \\
\left(\left(D_{\xi} \partial_{x} \beta_{p i} \circ P_{p i}^{\prime} \circ \varphi\right) u,\left(P_{p i}^{\prime} \circ \varphi\right) u\right)=\left(\left(D_{\xi} \partial_{x} \beta_{q j} \circ P_{q j}^{\prime} \circ \varphi\right) u,\left(P_{q j}^{\prime} \circ \varphi\right) u\right),
\end{array}\right.
$$

we have

$$
\left|I_{p i}-I_{q j}\right| \leq C\left(\sqrt{I_{p i}}+\sqrt{I_{q i}}+\|u\|_{m-2}\right)\|u\|_{m-2} .
$$

Here we have

$$
I_{q j} \leq C^{\prime}\left(I_{p i}+\|u\|_{m-2}^{2}\right) .
$$

Case (ii). It is sufficient to show that

$$
\begin{aligned}
& \sum_{k=1}^{N_{q j}}\left\|b_{k}^{(q j)}\left(D^{\prime}\right) P_{q j}^{\prime}(D) \varphi\left(D^{\prime}\right) u\right\|+\left\|P_{q j}(D) \varphi\left(D^{\prime}\right) u\right\| \\
& \quad \leq C\left(\left\|P_{p i}(D) \varphi\left(D^{\prime}\right) u\right\|+\left\|P_{p i^{\prime}}(D) \varphi\left(D^{\prime}\right) u\right\|+\|u\|_{m-2}\right) .
\end{aligned}
$$

Since for $X^{\prime} \in \widetilde{W}\left(X_{p}^{\prime}\right) \cap \widetilde{W}\left(X_{q}^{\prime}\right)$

$$
\begin{aligned}
P_{q j}^{\prime}(X) & =\frac{A_{0}(X)}{H_{q j}(X)}=\frac{A_{0}(X)}{h_{p i}(X) h_{p i^{\prime}}(X)} \\
& =\frac{A_{0}(X)}{\left(\tau-\alpha_{p i}\left(X^{\prime}\right)\right)\left(\tau-\alpha_{p i^{\prime}}\left(X^{\prime}\right)\right)}
\end{aligned}
$$




$$
\begin{gathered}
=\frac{1}{\alpha_{p i}\left(X^{\prime}\right)-\alpha_{p i^{\prime}}\left(X^{\prime}\right)}\left(P_{p i}(X)-P_{p i^{\prime}}(X)\right) \\
P_{q j}(X)=\left(\tau-\alpha_{q j}\left(X^{\prime}\right)\right) \frac{A_{0}(X)}{H_{q j}(X)}=\frac{\left(\tau-\alpha_{q j}\left(X^{\prime}\right)\right) A_{0}(X)}{\left(\tau-\alpha_{p i}\left(X^{\prime}\right)\right)\left(\tau-\alpha_{p i^{\prime}}\left(X^{\prime}\right)\right)} \\
=\frac{\alpha_{p i}\left(X^{\prime}\right)-\alpha_{q j}\left(X^{\prime}\right)}{\alpha_{p i}\left(X^{\prime}\right)-\alpha_{p i^{\prime}}\left(X^{\prime}\right)} P_{p i}(X)+\frac{\alpha_{p i^{\prime}}\left(X^{\prime}\right)-\alpha_{p j}\left(X^{\prime}\right)}{\alpha_{p i^{\prime}}\left(X^{\prime}\right)-\alpha_{p i}} P_{p i^{\prime}}(X),
\end{gathered}
$$

we have

$$
\begin{gathered}
\left\|b_{k}^{(q j)} P_{q j}^{\prime} \varphi u\right\|+\left\|P_{q j} \varphi u\right\| \leq\left\|b_{k}^{(q j)} \circ P_{q j}^{\prime} \circ \varphi u\right\|+\left\|P_{q j} \circ \varphi u\right\|+C\|u\|_{m-2} \\
\leq C^{\prime}\left\{\left\|P_{q j} \circ \varphi u\right\|+\left\|P_{p^{\prime}} \circ \varphi u\right\|+\|u\|_{m-2}\right\} \\
\leq C^{\prime \prime}\left\{\left\|P_{p i} \varphi u\right\|+\left\|P_{p i^{\prime}} \varphi u\right\|+\|u\|_{m-2}\right\} .
\end{gathered}
$$

Case (iii). It is sufficient to show that

$$
\left\|P_{q j} \varphi u\right\|+\left\|P_{q j^{j}}, \varphi u\right\| \leq C\left(\left\|P_{p_{i}} \varphi u\right\|+\sum_{k=1}^{N_{p i}}\left\|b_{k}^{(p i)} P_{p i}^{\prime} \varphi u\right\|+\|u\|_{m-2}\right) .
$$

Since for $X^{\prime} \in \widetilde{W}\left(X_{p}^{\prime}\right) \cap \widetilde{W}\left(X_{q}^{\prime}\right)$

$$
\begin{aligned}
P_{q j}(X) & =\frac{A_{0}(X)}{h_{q j}(X)}=\frac{h_{q j^{\prime}}(X) A_{0}(X)}{h_{q j^{\prime}}(X) h_{q j}(X)}=\left(\tau-\alpha_{q j^{\prime}}\left(X^{\prime}\right)\right) P_{p i}^{\prime}(X) \\
& =P_{p i}(X)+\left(\alpha_{p i}\left(X^{\prime}\right)-\alpha_{q j^{\prime}}\left(X^{\prime}\right)\right) P_{p i}^{\prime}(X), \\
P_{q j^{\prime}}(X) & =\left(\tau-\alpha_{q j}\left(X^{\prime}\right)\right) P_{p i}^{\prime}(X)=P_{p i}(X)+\left(\alpha_{p i}\left(X^{\prime}\right)-\alpha_{q j}\left(X^{\prime}\right)\right) P_{p i}^{\prime}(X),
\end{aligned}
$$

we have

$$
\begin{gathered}
\left\|P_{q j} \varphi u\right\|+\left\|P_{q j} \varphi u\right\| \leq\left\|P_{q j} \circ \varphi u\right\|+\left\|P_{q j^{\circ}} \circ \varphi u\right\|+C\|u\|_{m-2} \\
\leq C^{\prime}\left(\left\|P_{p i} \circ \varphi u\right\|+\left\|P_{p i}^{\prime} \circ \varphi u\right\|_{1}+\|u\|_{m-2}\right) .
\end{gathered}
$$

On the other hand, since

$$
\left(\tau-\alpha_{q j}\right)\left(\tau-\alpha_{q j^{\prime}}\right)=\left(\tau-\alpha_{p i}\right)^{2}-\beta_{p i},
$$

we have $\beta_{p i}\left(X^{\prime}\right) \neq 0$ for $X^{\prime} \in \widetilde{W}\left(X_{p}^{\prime}\right) \cap \widetilde{W}\left(X_{q}^{\prime}\right)$, hence we have

$$
\left\|P_{p i}^{\prime} \circ \varphi u\right\|_{1} \leq C\left(\sum_{k=1}^{N_{p i}}\left\|b_{k}^{(p i)} \circ P_{p i}^{\prime} \circ \varphi u\right\|+\|u\|_{m-2}\right) .
$$

Case (iv). Since

$$
P_{q j}(X)=\frac{A_{0}(X)}{h_{q j}(X)}=\frac{A_{0}(X)}{h_{p i}(X)}=P_{p i}(X),
$$


we have

$$
\begin{aligned}
\left\|P_{q j} \varphi u\right\| & \leq\left\|P_{q j} \circ \varphi u\right\|+C\|u\|_{m-1}=\left\|P_{p i} \circ \varphi u\right\|+C\|u\|_{m-1} \\
& \leq\left\|P_{p i} \varphi u\right\|+C^{\prime}\|u\|_{m-1} .
\end{aligned}
$$

Now, we return to the problem of the localization of $A(D)$. Using the decomposition of unity $\left\{\varphi_{p}\left(X^{\prime}\right)\right\}_{p=1, \ldots, M}$, we have

$$
\begin{aligned}
\varphi_{p}\left(D^{\prime}\right) & A(D)-A^{(p)}(D) \varphi_{p}\left(D^{\prime}\right) \\
= & D_{\xi} \varphi_{p}\left(D^{\prime}\right) \circ \partial_{x} A_{0}(D)-D_{\xi} A_{0}(D) \circ \partial_{x} \varphi_{p}\left(D^{\prime}\right)+L_{p},
\end{aligned}
$$

where

$$
\left\|L_{p} u\right\| \leq C\left\|(\Lambda+1)^{-1} u\right\|_{m-1} .
$$

Let $\psi_{p}\left(X^{\prime}\right)$ be smooth and homogeneous of degree 0 , whose support is in $\widetilde{W}\left(X_{p}^{\prime}\right)$, and $\phi_{p}\left(X^{\prime}\right)=1$ on the support of $\varphi_{p}\left(X^{\prime}\right)$. Then we have

$$
\begin{gathered}
\left\|\left(D_{\xi} \varphi_{p} \circ \partial_{x} A_{0}-D_{\xi} A_{0} \circ \partial_{x} \varphi_{p}\right) u\right\| \leq C\left\|\boldsymbol{P}^{(p)} \circ \psi_{p} u\right\| \\
\leq C^{\prime}\left\{\left\|\boldsymbol{P}^{(p)} \psi_{p} u\right\|+\|u\|_{m-2}\right\} .
\end{gathered}
$$

Moreover, we have from Lemma 5. 1

$$
\begin{aligned}
\left\|\boldsymbol{P}^{(p)} \psi_{p} u\right\|= & \left\|\boldsymbol{P}^{(p)} \psi_{p} \sum_{q=1}^{M} \varphi_{q} u\right\| \leq \sum_{q=1}^{M}\left\|\boldsymbol{P}^{(p)} \psi_{p} \circ \varphi_{q} u\right\|+C\|u\|_{m-2} \\
& \leq C^{\prime}\left(\sum_{q=1}^{M}\left\|\boldsymbol{P}^{(q)} \psi_{p} \circ \varphi_{q} u\right\|+\|u\|_{m-2}\right) \\
& \leq C^{\prime \prime}\left(\sum_{q=1}^{M}\left\|\boldsymbol{P}^{(q)} \varphi_{q} u\right\|+\|u\|_{m-2}\right)
\end{aligned}
$$

Hence we have

Lemma 5. 2 (Localization of A).

$$
\left\|A^{(p)} \varphi_{p} u\right\| \leq C\left\{\left\|\varphi_{p} A u\right\|+\sum_{q=1}^{M}\left\|\boldsymbol{P}^{(q)} \varphi_{q} u\right\|+\left\|(\Lambda+1)^{-1} u\right\|_{m-1}\right\} .
$$

Now we apply Proposition 4.3 and its corollary to localized operators $A^{(p)}$, then we have for $\gamma>\gamma_{0}$

$$
e^{-2 \gamma t}\left\{\left\|\boldsymbol{P}^{(p)} \varphi_{p} u(t)\right\|^{2}+\sum_{k=0}^{m-2} \gamma^{2(m-1-k)}\left\|\varphi_{p} u(t)\right\|_{k}^{2}+\gamma^{2}\left\|(\Lambda+\gamma)^{-1} \varphi_{p} u(t)\right\|_{m-1}^{2}\right\}
$$




$$
\begin{gathered}
+\gamma \int_{0}^{t} e^{-2_{i} t}\left\{\left\|\mathbb{P}^{(p)} \varphi_{p} u(t)\right\|^{2}+\sum_{k=0}^{m-2} \gamma^{2(m-1-k)}\left\|\varphi_{p} u(t)\right\|_{k}^{2}\right. \\
\left.+\gamma^{2}\left\|(\Lambda+\gamma)^{-1} \varphi_{p} u(t)\right\|_{m-1}^{2}\right\} d t \\
\leq C\left\{\left\|\boldsymbol{P}^{(p)} \varphi_{p} u(0)\right\|^{2}+\sum_{k=0}^{m-2} \gamma^{2(m-1-k)}\left\|\varphi_{p} u(0)\right\|_{k}^{2}\right. \\
\left.+\frac{1}{\gamma} \int_{0}^{t} e^{-2 \gamma t}\left\|A^{(p)} \varphi_{p} u(t)\right\|^{2} d t\right\} \\
\leq C^{\prime}\left\{\left\|\boldsymbol{P}^{(p)} \varphi_{p} u(0)\right\|^{2}+\sum_{k=0}^{n-2} \gamma^{2(m-1-k)}\left\|\varphi_{p} u(0)\right\|_{k}^{2}\right. \\
+\frac{1}{\gamma} \int_{0}^{t} \mathcal{C}^{-2 \gamma t}\left(\left\|\varphi_{p} A u(t)\right\|^{2}+\sum_{q=1}^{M}\left\|\mathbb{P}^{(q)} \varphi_{q} u(t)\right\|^{2}\right. \\
\left.\left.+\left\|(\Lambda+1)^{-1} u(t)\right\|_{m-1}^{2}\right) d t\right\} .
\end{gathered}
$$

Summing them up with respect to $p$, we have

Theorem $\mathbb{1}$. Let us assume Conditions $(A)$ and $(B)$, then there exist $\gamma_{0}>0$ and $C>0$ such that for $\gamma>\gamma_{0}$

$$
\begin{gathered}
e^{-2 \gamma t}\left\{\|\mathbb{P} u(t)\|^{2}+\sum_{k=0}^{m-2} \gamma^{2(m-1-k)}\|u(t)\|_{k}^{2}+\gamma^{2}\left\|(\Lambda+\gamma)^{-1} u(t)\right\|_{m-1}^{2}\right\} \\
+\gamma \int_{0}^{t} e^{-2 \gamma t}\left\{\|\mathbb{P} u(t)\|^{2}+\sum_{k=0}^{m-2} \gamma^{2(m-1-k)}\|u(t)\|_{k}^{2}\right. \\
\left.+\gamma^{2}\left\|(\Lambda+\gamma)^{-1} u(t)\right\|_{m-1}^{2}\right\} d t \\
\leq C\left\{\|\mathbb{P} u(0)\|^{2}+\sum_{k=0}^{m-2} \gamma^{2(m-1-k)}\|u(0)\|_{k}^{2}+\frac{1}{\gamma} \int_{0}^{t} e^{-2 \tau t}\|A u(t)\|^{2} d t\right\},
\end{gathered}
$$

where

$$
\|\mathbb{P} u\|^{2}=\sum_{p=1}^{M}\left\|\boldsymbol{P}^{(p)} \varphi_{p} u\right\|^{2}
$$

Remark Let us denote

$$
\|u\|_{l, r}^{2}=\sum_{l=0}^{k} \gamma^{2(k-l)}\|u\|_{l}^{2}
$$

then 


$$
c_{1}\left\|(\Lambda+\gamma)^{-1} u\right\|_{m-1, r}^{2} \leq\|u\|_{m-2, \gamma}^{2}+\left\|(\Lambda+\gamma)^{-1} u\right\|_{m-1}^{2} \leq c_{2}\left\|(\Lambda+\gamma)^{-1} u\right\|_{m-1, \gamma}^{2} .
$$

\section{Corollary. Let}

$$
\int_{-\infty}^{\infty} e^{-2 \tau t}\|u(t)\|_{m}^{2} d t<+\infty
$$

then

$$
\int_{-\infty}^{\infty} e^{-2 \gamma t}\left\|(\Lambda+\gamma)^{-1} u(t)\right\|_{m-1, r}^{2} \leq \frac{C}{\gamma^{4}} \int_{-\infty}^{\infty} e^{-2 r t}\|A u(t)\|^{2} d t
$$

Finally, we consider energy inequalities of higher order or lower order.

\section{Lemma 5. 3.}

$$
\begin{gathered}
\left\|\left(D_{\xi} A_{0}\right)(D) u\right\|+\left\|(\Lambda+1)^{-1}\left(D_{x} A_{0}\right)(D) u\right\| \\
\leq C\left\{\|\boldsymbol{P} u\|+\left\|(\Lambda+1)^{-1} u\right\|_{m-1}\right\} .
\end{gathered}
$$

Proof. We have

$$
\begin{aligned}
\left\|\left(D_{\xi} A_{0}\right)(D) u\right\| & \leq \sum_{p=1}^{M}\left\|\left(D_{\xi} A_{0}\right)(D) \varphi_{p}\left(D^{\prime}\right) u\right\| \\
& \leqq \sum_{p=1}^{M}\left\|\left(D_{\xi} A_{0}^{(p)}\right)(D) \varphi_{p}\left(D^{\prime}\right) u\right\|+C\left\|(\Lambda+1)^{-1} u\right\|_{m-1},
\end{aligned}
$$

and

$$
\begin{gathered}
\left\|(\Lambda+1)^{-1}\left(D_{x} A_{0}\right)(D) u\right\| \leq \sum_{p=1}^{M}\left\|(\Lambda+1)^{-1}\left(D_{x} A_{0}\right)(D) \varphi_{p}\left(D^{\prime}\right) u\right\| \\
\leqq \sum_{p=1}^{M}\left\|(\Lambda+1)^{-1}\left(D_{x} A_{0}^{(p)}\right)(D) \varphi_{p}\left(D^{\prime}\right) u\right\| \\
+C\left\|(\Lambda+1)^{-1} u\right\|_{m-1} .
\end{gathered}
$$

On the other hands, we have

$$
\begin{aligned}
& \left(D_{\xi} A_{0}^{(p)}\right)\left(\alpha_{p i}\left(X^{\prime}\right), X^{\prime}\right) \equiv 0 \quad \bmod \boldsymbol{b}^{(p i)}\left(X^{\prime}\right), \\
& \left(D_{x} A_{0}^{(p)}\right)\left(\alpha_{p i}\left(X^{\prime}\right), X^{\prime}\right) \equiv 0 \bmod \boldsymbol{b}^{(p i)}\left(X^{\prime}\right),
\end{aligned}
$$

then we have 


$$
\begin{gathered}
\left\|D_{\xi} A_{0}^{(p)}(D) v\right\|+\left\|(\Lambda+1)^{-1}\left(D_{x} A_{0}^{(p)}\right)(D) v\right\| \\
\leq C\left(\left\|\mathbb{P}^{(p)} v\right\|+\|v\|_{m-2}\right) .
\end{gathered}
$$

Now, we remark

$$
\left\|\left(D_{x} A(D)-A(D) D_{x}\right) u\right\|=\left\|\left(D_{x} A\right)(D) u\right\| \leq C\left\{\|\mathbb{P} \Lambda u\|+\|u\|_{m-1}\right\},
$$

and we apply Theorem I, replacing $D_{x} u$ instead of $u$, then we have

$$
\begin{aligned}
& \mathcal{c}^{-2 r t}\left(\left\|\boldsymbol{P} D_{x} u(t)\right\|^{2}+\gamma^{2}\left\|D_{x} u(t)\right\|_{m-2, r}^{2}\right) \\
& +r \int_{0}^{t} e^{-2 r t}\left(\left\|\mathbb{P} D_{x} u(t)\right\|^{2}+\gamma^{2}\left\|D_{x} u(t)\right\|_{m-2, r}^{2}\right) d t \\
& \leq C\left\{\|\left(\boldsymbol{P} D_{x} u(0)\left\|^{2}+\gamma^{2}\right\| D_{x} u(0) \|_{m-?, r}^{2}\right.\right. \\
& \left.+\frac{1}{r} \int_{0}^{t} e^{-2 \gamma t}\left(\left\|D_{x}(A u)(t)\right\|^{2}+\|\mathbb{P} \Lambda u(t)\|^{2}+\|u(t)\|_{m-1}^{2}\right) d t\right\} .
\end{aligned}
$$

Therefore, together with Theorem I, we have for large $\gamma$

$$
\begin{aligned}
& e^{-2 r t}\left\{\|\boldsymbol{P} u(t)\|_{1}^{2}+\gamma^{2}\|u(t)\|_{m-1, \gamma}^{2}\right\} \\
& \quad+\gamma \int_{0}^{t} e^{-2 \gamma t}\left\{\|\mathbb{P} u(t)\|_{1}^{2}+\gamma^{2}\|u(t)\|_{m-1, \gamma}^{2}\right\} d t \\
& \leq C\left\{\|\boldsymbol{P} u(0)\|_{1}^{2}+\gamma^{2}\|u(0)\|_{m-1, r}^{2}+\frac{1}{r} \int_{0}^{t} e^{-2 \gamma t}\|A u(t)\|_{1}^{2} d t\right\} .
\end{aligned}
$$

Then we have step by step

Theorem $\mathbb{H}^{\prime}$. Let us assume Conditions $(A)$ and $(B)$, then there exist $\gamma_{k}>0$ and $C_{k}>0$ such that for $\gamma>\gamma_{k}$

$$
\begin{gathered}
e^{-2 \gamma t}\left\{\|\boldsymbol{P} u(t)\|_{k}^{2}+\gamma^{2}\|u(t)\|_{m-2+k, \gamma}^{2}\right\} \\
+\gamma \int_{0}^{t} e^{-2 \gamma t}\left\{\|\mathbb{P} u(t)\|_{k}^{2}+\gamma^{2}\|u(t)\|_{m-2+k, \gamma}^{2}\right\} d t \\
\leq C_{k}\left\{\|\boldsymbol{P} u(0)\|_{k}^{2}+\gamma^{2}\|u(0)\|_{m-2+k, r}^{2}+\frac{1}{\gamma} \int_{0}^{t} e^{-2 \gamma t}\|A u(t)\|_{k}^{2} d t\right\} \\
(k=0,1,2, \cdots) .
\end{gathered}
$$

Lemma 5.4. 


$$
\begin{aligned}
& \left\|\left\{(\Lambda+\gamma)^{-1} A-A(\Lambda+\gamma)^{-1}\right\} u\right\| \\
& \quad \leq C\left\{\left\|\boldsymbol{P}(\Lambda+\gamma)^{-1} u\right\|+\left\|(\Lambda+\gamma)^{-2} u\right\|_{m-1}\right\} .
\end{aligned}
$$

Proof. We have

$$
\begin{aligned}
(\Lambda+\gamma)^{-1} A-A(\Lambda+\gamma)^{-1}=(\Lambda+\gamma)^{-1}(A \Lambda-\Lambda A)(\Lambda+\gamma)^{-1} \\
=(\Lambda+\gamma)^{-1}\left(-D_{\xi} \Lambda \cdot \partial_{x} A_{0}+\{\{m-1\}\}\right)(\Lambda+\gamma)^{-1} .
\end{aligned}
$$

From Lemma 5. 3, we have

$$
\begin{aligned}
\|(\Lambda+\gamma)^{-1} & \left(D_{\xi} \Lambda\right)\left(\partial_{x} A_{0}\right)(\Lambda+\gamma)^{-1} u \| \\
& \leq C\left\|(\Lambda+\gamma)^{-1}\left(\partial_{x} A_{0}\right)(\Lambda+\gamma)^{-1} u\right\| \\
& \leq C^{\prime}\left(\left\|\boldsymbol{P}(\Lambda+\gamma)^{-1} u\right\|+\left\|(\Lambda+\gamma)^{-2} u\right\|_{m-1}\right) . \quad \text { Q.E.D. }
\end{aligned}
$$

Now we apply Theorem I, replacing $(\Lambda+\gamma)^{-1} u$ instead of $u$, then we have

$$
\begin{aligned}
& e^{-2 \tau t}\left\{\left\|\boldsymbol{P}(\Lambda+\gamma)^{-1} u(t)\right\|^{2}+\gamma^{2}\left\|(\Lambda+\gamma)^{-2} u(t)\right\|_{m-1, \gamma}^{2}\right\} \\
& +\gamma \int_{0}^{t} e^{-2 \gamma t}\left\{\left\|\boldsymbol{P}(\Lambda+\gamma)^{-1} u(t)\right\|^{2}+\gamma^{2}\left\|(\Lambda+\gamma)^{-2} u(t)\right\|_{m-1, \gamma}^{2}\right\} d t \\
& \leq C\left\{\left\|\boldsymbol{P}(\Lambda+\gamma)^{-1} u(0)\right\|^{2}+\gamma^{2}\left\|(\Lambda+\gamma)^{-2} u(0)\right\|_{m-1, \gamma}^{2}\right\} \\
& +\frac{1}{\gamma} \int_{0}^{t} e^{-2 \gamma t}\left\{\left\|(\Lambda+\gamma)^{-1} A u(t)\right\|^{2}+\left\|\boldsymbol{P}(\Lambda+\gamma)^{-1} u(t)\right\|^{2}\right. \\
& \left.+\left\|(\Lambda+\gamma)^{-2} u(t)\right\|_{m-1}^{2}\right\} d t .
\end{aligned}
$$

Hence we have for large $\gamma$

$$
\begin{aligned}
e^{-2 \gamma t} \| \boldsymbol{P} & (\Lambda+\gamma)^{-1} u(t)\left\|^{2}+\gamma^{2}\right\|(\Lambda+\gamma)^{-2} u(t) \|_{m-1, \gamma}^{2} \\
& +\gamma \int_{0}^{t} e^{-2 \gamma t}\left\{\left\|\boldsymbol{P}(\Lambda+\gamma)^{-1} u(t)\right\|^{2}+\gamma^{2}\left\|(\Lambda+\gamma)^{-2} u(t)\right\|_{m-1, \gamma}^{2}\right\} d t \\
\leq & C\left\{\left\|\boldsymbol{P}(\Lambda+\gamma)^{-1} u(0)\right\|^{2}+\left\|(\Lambda+\gamma)^{-2} u(0)\right\|_{m-1, \gamma}^{2}\right. \\
& \left.+\frac{1}{\gamma} \int_{0}^{t} e^{-2 r t}\left\|(\Lambda+\gamma)^{-1} A u(t)\right\|^{2} d t\right\} .
\end{aligned}
$$

In this way, we have step by step 
Theorem 1". Let us assume Conditions $(A)$ and $(B)$, then there exist $\gamma_{k}>0$ and $C_{k}>0$ such that for $r>\gamma_{k}$

$$
\begin{aligned}
& e^{-2 \gamma t}\left\{\left\|\mathbb{P}(\Lambda+\gamma)^{-k} u(t)\right\|^{2}+\gamma^{2}\left\|(\Lambda+\gamma)^{-1-k} u(t)\right\|_{m-1, \gamma}^{2}\right\} \\
& \quad+\gamma \int_{0}^{t} e^{-2 \gamma t}\left\{\left\|\mathbb{P}(\Lambda+\gamma)^{-k} u(t)\right\|^{2}+\gamma^{2}\left\|(\Lambda+\gamma)^{-1-k} u(t)\right\|_{m-1, \gamma}^{2}\right\} d t \\
& \leq C_{k}\left\{\left\|\mathbb{P}(\Lambda+\gamma)^{-k} u(0)\right\|^{2}+\gamma^{2}\left\|(\Lambda+\gamma)^{-1-k} u(0)\right\|_{m-1, \gamma}^{2}\right. \\
& \left.\quad+\frac{1}{\gamma} \int_{0}^{t} e^{-2 \gamma t}\left\|(\Lambda+\gamma)^{-k} A u(t)\right\|^{2} d t\right\} \quad(k=0,1,2, \cdots) .
\end{aligned}
$$

\section{$\S 6 . \quad$ Existence Theorems}

First, we consider a slight variation of energy inequalities. Let $\alpha(t)$ be a smooth function of $t$ such that

$$
\begin{cases}\alpha(t)=1 & \text { for } t \geq 1 \\ \alpha(t)=0 & \text { for } t \leq-1\end{cases}
$$

and

$$
0<\alpha(t)<1 \quad \text { for } \quad-1<t<1 \text {. }
$$

Let us denote a weight function

$$
e_{\delta}(t)=\alpha(t)+\alpha(-t) e^{\delta t}
$$

for $|\delta|<1$, then we have

$$
\left|\left(\frac{d}{d t}\right)^{j} e_{\delta}(t)\right| \leq C_{j} e_{\delta}(t)
$$

Hence, let us denote

$$
\frac{e_{\delta}^{\prime}(t)}{e_{\delta}(t)}=\psi_{\delta}(t)
$$

then we have

$$
e_{\delta}(t) D_{t} u=\left(D_{t}+i \psi_{\delta}(t)\right) e_{\delta}(t) u
$$

therefore we have

$$
e_{\delta}(t) A\left(D_{t}, D_{x}^{\prime}\right) u=A\left(D_{t}+i \psi_{\delta}(t), D_{x}^{\prime}\right) e_{\delta}(t) u=\tilde{A}\left(D_{t}, D_{x}^{\prime}\right) e_{\delta}(t) u
$$


Since

$$
\tilde{A}_{0}(X)=A_{0}(X), \quad \tilde{A}_{1}(X)=A_{1}(X)+\partial_{\tau} A_{0}(X) i \psi_{\delta}(t)
$$

we have

Lemma 6. 1. Let Conditions $(A)$ and $(B)$ be satisfied by $A(D)$, then they are satisfied also by $\tilde{A}(D)$.

Now let us apply Corollary of Theorem I on $\tilde{A}$, then we have

$$
\int_{-\infty}^{\infty} e^{-2 \gamma t}\left\|(\Lambda+\gamma)^{-1}\left(e_{\delta} u\right)(t)\right\|_{m-1, \gamma}^{2} d t \leq \frac{C}{\gamma^{4}} \int_{-\infty}^{\infty} e^{-2 \gamma t} \tilde{A}\left\|\left(e_{\delta} u\right)(t)\right\|^{2} d t
$$

Since

$$
\begin{gathered}
\left\|(\Lambda+\gamma)^{-1} e_{\delta} u\right\|_{m-1, r}^{2} \geq c\left\{\left\|(\Lambda+\gamma)^{-1} D_{t}^{m-1}\left(e_{\delta} u\right)\right\|^{2}\right. \\
\left.+\sum_{k=0}^{m-2} \gamma^{2(m-2-k)}\left\|D_{t}^{k}\left(e_{\delta} u\right)\right\|^{2}\right\} \\
=c e_{\delta}^{2}\left\{\left\|(\Lambda+\gamma)^{-1}\left(D_{t}-i \psi_{\delta}\right)^{m-1} u\right\|^{2}+\sum_{k=0}^{m-2} \gamma^{2(m-2-k)}\left\|\left(D_{t}-i \psi_{\delta}\right)^{k} u\right\|^{2}\right\} \\
\geq c e_{\delta}^{2}\left\{\left\|(\Lambda+\gamma)^{-1} D_{t}^{m-1} u\right\|^{2}+\sum_{k=0}^{m-2} \gamma^{2(m-2-k)}\left\|D_{t}^{k} u\right\|^{2}\right. \\
\left.-C \frac{1}{\gamma^{2}} \sum_{k=0}^{m-2} \gamma^{2(m-2-k)}\left\|D_{t}^{k} u\right\|^{2}\right\}
\end{gathered}
$$

we have for large $\gamma$

$$
\int_{-\infty}^{\infty} e^{-2 \gamma t} e_{\delta}(t)^{2}\left\|(\Lambda+\gamma)^{-1} u(t)\right\|_{m-1, \gamma}^{2} d t \leq \frac{C}{\gamma^{4}} \int_{-\infty}^{\infty} e^{-2 \tau t} e_{\delta}(t)^{2}\|A u(t)\|^{2} d t
$$

Let us denote

$$
e_{r, r^{\prime}}(t)=\alpha(t) e^{r t}+\alpha(-t) e^{r^{\prime} t}
$$

for $\left|\gamma-\gamma^{\prime}\right| \leq 1$, then

$$
e_{\gamma, \gamma^{\prime}}(t)=e^{r t} e_{\delta}(t) \quad\left(\delta=\gamma^{\prime}-\gamma\right)
$$

Hence we have

Proposition 6. 2. Let $\gamma, \gamma^{\prime} \geq \gamma_{0}$ and $\left|\gamma-\gamma^{\prime}\right| \leq 1$, then

$$
\int_{-\infty}^{\infty} e_{-r_{,}-r^{\prime}}(t)^{2}\|u(t)\|_{m-2, r}^{2} d t \leq \frac{C}{\gamma^{4}} \int_{-\infty}^{\infty} e_{-r_{,}-r^{\prime}}(t)^{2}\|A u(t)\|^{2} d t
$$


Corollary. Let $\gamma, \gamma^{\prime} \geq \gamma_{0},\left|\gamma-\gamma^{\prime}\right| \leq 1$, and

$$
\int_{-\infty}^{\infty} e^{-2 \gamma t}\|u(t)\|_{m}^{2} d t<+\infty, \quad \int_{-\infty}^{\infty} e^{-2 r^{\prime} t}\|v\|_{m}^{2} d t<+\infty .
$$

Then $A u=A v$ implies $u=v$.

Proof. Let $w=u-v$, then we have $A w=0$ and

$$
\int_{-\infty}^{\infty} e_{-r,-r^{\prime}}(t)^{2}\|w(t)\|_{m}^{2} d t<+\infty
$$

Then we have from Proposition 6.2

$$
\int_{-\infty}^{\infty} e_{-r, r^{\prime}}(t)^{2}\|w(t)\|_{m-2, r}^{2} d t \leq \frac{C}{\gamma^{4}} \int_{-\infty}^{\infty} e_{-r,-r^{\prime}}(t)^{2}\|A w(t)\|^{2} d t=0
$$

Q.E.D.

Next, let us consider the formal adjoint $A^{*}$ of $A$. Since

$$
A^{*}(D)=A_{0}(D)+\left\{\bar{A}_{1}(D)+\left(D_{\xi} \partial_{x} A_{0}\right)(D)\right\}+\{\{m-2\}\},
$$

we have

$$
\begin{aligned}
\left(A^{*}\right)_{0}(X) & =A_{0}(X), \\
L_{A^{*}}(X) & =\overline{A_{1}(X)}+D_{\xi} \partial_{x} A_{0}(X)-\frac{1}{2} D_{\xi} \partial_{x} A_{0}(X) \\
= & \overline{A_{1}(X)-\frac{1}{2} D_{\xi} \partial_{x} A_{0}(X)}=\overline{L_{A}(X) .}
\end{aligned}
$$

Here we have

Lemma 6. 3. Let Conditions $(A)$ and $(B)$ be satisfied by $A(D)$, then they are satisfied also by $A^{*}(D)$.

Hence, we have energy inequalities for $A^{*}(D)$, corresponding to Theorems I, I', I" for $A(D)$. Therefore, in usual technique, ([5])

Theorem III. Let us assume Conditions $(A)$ and $(B)$ for $A(D)$. Then Cauchy problem:

$$
\left\{\begin{array}{l}
A(D) u=f\left(t, x^{\prime}\right) \quad \text { in }\left\{t>0, x^{\prime} \in R^{n}\right\}, \\
\left.D_{t}^{j} u\right|_{t=0}=u_{j}\left(x^{\prime}\right) \quad(j=0,1, \cdots, m-1) \text { in }\left\{x^{\prime} \in \mathbb{R}^{n}\right\}
\end{array}\right.
$$


is well posed in sense of Sobolev's space.

\section{Chapter III. Finiteness of Propagation Speed}

\section{$\S 7$. Change of Variables}

Let us consider basic properties about the change of differential operators caused by a change of variables. Let us denote

$$
\left(t, x^{\prime}\right)=x=\left(x_{0}, x_{1}, \cdots, x_{n}\right), \quad\left(\tau, \xi^{\prime}\right)=\xi=\left(\xi_{0}, \xi_{1}, \cdots, \xi_{n}\right),
$$

and let $x=\varphi(y)$ be a change of variables $x$ into $y$, and $y=\phi(x)$ be its inverse. Then we have

$$
\begin{aligned}
& \partial_{y_{i}}=\sum_{j=0}^{n} \varphi_{j(i)}(y) \partial_{x_{j}}\left(\partial_{y}={ }^{t} \Phi(y) \partial_{x}\right), \\
& \partial_{x_{i}}=\sum_{j=0}^{n} \psi_{j(i)}(x) \partial_{y_{j}}\left(\partial_{x}={ }^{t} \Psi(x) \partial_{y}\right),
\end{aligned}
$$

where

$$
\begin{aligned}
& \Phi(y)=\left(\varphi_{i(j)}(y)\right)_{i, j=0, \ldots, n}=\left(\partial_{y_{j}} \varphi_{i}(y)\right)_{i, j=0, \ldots, n}, \\
& \Psi(x)=\left(\psi_{i(j)}(x)\right)_{i, j=0, \ldots, n}=\left(\partial_{x_{j}} \psi_{i}(x)\right)_{i, j=0, \ldots, n},
\end{aligned}
$$

and

$$
\Phi(\psi(x)) \Psi(x)=I
$$

Hence we have another change of variables $X=(x, \xi)$ into $Y=(y, \eta)$ and its inverse:

$$
X(Y)=\left(\varphi(y),{ }^{t} \Psi(\varphi(y)) \eta\right), \quad Y(X)=\left(\psi(x),{ }^{t} \Phi(\psi(x)) \xi\right) .
$$

Then we have

$$
\left\{\begin{aligned}
\partial_{x_{i}} & =\sum_{j=0}^{n} \phi_{j(i)}(x) \partial_{y_{j}}+\sum_{j, k, l=0}^{n} \xi_{k} \varphi_{k(l, j)}(y) \psi_{l(i)}(x) \partial_{\eta_{j}} \\
& =\sum_{j=0}^{n} \phi_{j(i)}(x) \partial_{y_{j}}-\sum_{j, k, l=0}^{n} \eta_{k} \psi_{k(i, l)}(x) \varphi_{l(j)}(y) \partial_{\eta_{j}}, \\
\partial_{\xi_{i}} & =\sum_{j=0}^{n} \varphi_{i(j)}(y) \partial_{\eta_{j}},
\end{aligned}\right.
$$

that is, 


$$
\left(\begin{array}{c}
\partial_{x} \\
\partial_{\xi}
\end{array}\right)=\left(\begin{array}{cc}
{ }^{t} \Psi(\varphi(y)) & -\sum_{k=0}^{n} \eta_{k} H_{\psi_{k}}(\varphi(y)) \Phi(y) \\
0 & \Phi(y)
\end{array}\right)\left(\begin{array}{l}
\partial_{y} \\
\partial_{\eta}
\end{array}\right),
$$

where

$$
\begin{aligned}
& H_{\varphi_{k}}(y)=\left(\varphi_{k(i, j)}(y)\right)_{i, j=0, \cdots, n}=\left(\partial_{y_{i}} \partial_{y_{j}} \varphi_{k}(y)\right)_{i, j=0, \ldots, n}, \\
& H_{\psi_{k}}(x)=\left(\psi_{k(i, j)}(x)\right)_{i, j=0, \cdots, n}=\left(\partial_{x_{i}} \partial_{x_{j}} \psi_{k}(x)\right)_{i, j=0 \ldots, n},
\end{aligned}
$$

and

$$
{ }^{t} \Psi H_{\varphi_{h}} \Psi=-\sum_{l=0}^{n} \varphi_{k(l)} H_{\psi_{l}}
$$

Hence we have

$$
\begin{gathered}
\partial_{\xi} a(X) \cdot \partial_{x} b(X)=\left(\Phi \partial_{\eta} a\right) \cdot\left({ }^{t} \Psi \partial_{y} b-\sum_{k=0}^{n} \eta_{k} H \psi_{\psi_{l}} \Phi \partial_{\eta} b\right) \\
=\partial_{\eta} a \cdot \partial_{y} b-\left(\Phi \partial_{\eta} a\right) \cdot\left(\sum_{k=0}^{n} \eta_{k} H_{\psi_{k}} \Phi \partial_{\eta} b\right) \\
=\partial_{\eta} a \cdot \partial_{y} b-\partial_{\xi} a \cdot\left(\sum_{k=0}^{n} \eta_{k} H_{\psi_{k}} \partial_{\xi} b\right),
\end{gathered}
$$

therefore we have

Lemma 7. 1. Let $a=a(X)$ and $b=b(X)$, then

$$
\begin{array}{r}
\partial_{\xi} a(X) \cdot \partial_{x} b(X)-\partial_{\eta} a(X(Y)) \cdot \partial_{y} b(X(Y)) \\
=-\partial_{\xi} a(X) \cdot\left(\sum_{k=0}^{n} \eta_{k} H_{\psi_{k}}(x) \partial_{\xi} b(X)\right),
\end{array}
$$

and moreover

$$
\left\{a(X), b\left(X^{\top}\right)\right\}_{X}=\{a(X(Y)), b(X(Y))\}_{Y} .
$$

Now, let us consider

$$
\mathcal{A}\left(y, D_{y}\right)=A\left(\varphi(y),{ }^{t} \Psi(\varphi(y)) D_{y}\right) .
$$

We denote

$$
\mathcal{A}(Y)=\mathscr{A}_{0}(Y)+\bar{A}_{1}(Y)+\cdots, \quad Y=(y, \eta),
$$

where $\mathcal{A}_{j}(Y)$ is homogeneous of degree $m-j$ with respect to $\eta$, and we denote 


$$
L_{A}(Y)=\mathscr{A}_{1}(Y)-\frac{1}{2} D_{\eta} \partial_{y} \mathscr{A}_{0}(Y)
$$

then we have

\section{Lemma 7. 2.}

$$
\mathscr{A}_{0}(Y)=A_{0}(X(Y))
$$

and

$$
\begin{aligned}
\mathscr{A}_{1}(Y)=A_{1}(X(Y)) & +\frac{1}{2}\left\{D_{\eta} \partial_{y} A_{0}(X(Y))-\left(D_{\xi} \partial_{x} A_{0}\right)(X(Y))\right\} \\
& +\sum_{i, k=0}^{n} \partial_{x_{i}}\left\{\varphi_{i(k)}(\psi(x))\right\} D_{\eta_{k}} A_{0}(X(Y)),
\end{aligned}
$$

that is,

$$
L_{\mathfrak{A}}(Y)-L_{A}(X(Y))=\frac{1}{2} \sum_{i, k=0}^{n} \partial_{x_{i}}\left\{\varphi_{i(k)}(\psi(x))\right\} D_{\eta_{k}} A_{0}(X(Y)) .
$$

Proof Let us denote

$$
A_{0}(X)=\sum_{i_{1}=0}^{n} \cdots \sum_{i_{m}=0}^{n} a_{i_{1} \cdots i_{m}}(x) \xi_{i_{1} \ldots \xi_{i_{m}}}
$$

where $a_{i_{1} \ldots i_{m}}$ are invariant with respect to permutations of suffixes. Now we consider

$$
A_{0}\left(\varphi(y),{ }^{t} \Psi(\varphi(y)) D_{y}\right)=\sum_{i_{1}, \cdots, i_{m}} a_{i_{1} \cdots i_{m}}(\varphi(y)) \Xi_{i_{1}}\left(y, D_{y}\right) \cdots \Xi_{i_{m}}\left(y, D_{y}\right),
$$

where

$$
\boldsymbol{\Xi}\left(y, D_{y}\right)={ }^{t} \Psi(\varphi(y)) D_{y},
$$

then we have

$$
\begin{gathered}
A_{0}\left(\varphi(y),{ }^{t} \Psi(\varphi(y)) D_{y}\right)=\sum a_{i_{1} \cdots i_{m}}(\varphi(y)) \Xi_{i_{1}}\left(y, D_{y}\right) \circ \cdots \circ \Xi_{i_{m}}\left(y, D_{y}\right) \\
+\frac{m(m-1)}{2} \sum a_{i_{1} \cdots i_{m}}(\varphi(y))\left(D_{\eta} \Xi_{i_{1}}\right)\left(y, D_{y}\right) \circ\left(\partial_{y} \Xi_{i_{2}}\right)\left(y, D_{y}\right) \\
\circ \Xi_{i_{3}}\left(y, D_{y}\right) \circ \cdots \circ \Xi_{i_{m}}\left(y, D_{y}\right)+\{\{m-2\}\}
\end{gathered}
$$

Since we have from Lemma 7.1

$$
D_{\eta} \boldsymbol{\Xi}_{i}(Y) \cdot \partial_{y} \boldsymbol{\Xi}_{j}(Y)
$$




$$
=D_{\xi} \xi_{i} \cdot \partial_{x} \xi_{j}+\frac{1}{i} \partial_{\xi} \xi_{i} \cdot\left(\sum_{k=0}^{n} \eta_{k} H_{\psi_{k}} \partial_{\xi} \xi_{j}\right)=\frac{1}{i} \sum_{k=0}^{n} \eta_{k} \psi_{k(i, j)}(x)
$$

we have

$$
\begin{gathered}
A_{0}\left(\varphi(y),{ }^{t} \Psi\left(\varphi(y) D_{y}\right)=\sum a_{i_{1} \cdots i_{m}}(\varphi(y)) \Xi_{i_{1}}\left(y, D_{y}\right) \circ \cdots \circ \Xi_{i_{m}}\left(y, D_{y}\right)\right. \\
+\frac{1}{i} \frac{m(m-1)}{2} \sum a_{i_{1} \cdots i_{m}}(\varphi(y)) \sum_{k=0}^{n} \phi_{k\left(i_{1}, i_{2}\right)}(\varphi(y)) D_{y_{k}} \\
\circ \Xi_{i_{s}}\left(y, D_{y}\right) \circ \cdots \circ \Xi_{i_{m}}\left(y, D_{y}\right)+\{\{m-2\}\} .
\end{gathered}
$$

Here we have

$$
\dot{A}_{0}(Y)=\sum a_{i_{1} \cdots i_{m}}(\varphi(y)) \Xi_{i_{1}}(Y) \cdots \Xi_{i_{m}}(Y)=A_{0}(X(Y)),
$$

and

$$
\begin{aligned}
& A_{1}(Y)-A_{1}(X(Y)) \\
& =\frac{1}{i} \frac{m(m-1)}{2} \sum_{i_{1}, \cdots, i_{m}} a_{i_{1} \cdots i_{m}}(\varphi(y)) \sum_{k=0}^{n} \eta_{k} \psi_{k\left(i_{1}, i_{2}\right)}(\varphi(y)) \Xi_{i_{3}}(Y) \cdots \Xi_{i_{m}}(Y) \\
& =\frac{1}{2 i} \sum_{i, j, k} \eta_{k} \psi_{k(i, j)}(\varphi(y))\left(\partial_{\xi_{i}} \partial_{\xi_{j}} A_{0}\right)(X(Y)) \\
& =\frac{1}{2 i} \sum_{i}\left\{\sum_{j} \psi_{j(i)}(x) \partial_{y_{j}}-\partial_{x_{i}}\right\} \partial_{\xi_{i}} A_{0} \\
& =\frac{1}{2 i}\left\{\sum_{i, j} \psi_{j(i)}(x) \partial_{y_{j}}\left(\sum_{k} \varphi_{i(k)}(y) \partial_{\eta_{k}} A_{0}\right)-\sum_{i} \partial_{x_{i}} \partial_{\xi_{i}} A_{0}\right\} \\
& =\frac{1}{2 i}\left\{\sum_{j} \partial_{y_{j}} \partial_{\eta_{j}} A_{0}+\sum_{i, j, k} \psi_{j(i)}(x) \varphi_{i(j, k)}(y) \partial_{\eta_{k}} A_{0}-\sum_{i} \partial_{x_{i}} \partial_{\xi_{i}} A_{0}\right\}
\end{aligned}
$$

where

$$
\begin{array}{r}
\sum_{i, j, k} \phi_{j(i)}(x) \varphi_{i(j, k)}(y) \partial_{\eta_{k}} A_{0}=\sum \partial_{x_{i}}\left\{\varphi_{i(k)}(\psi(x))\right\} \partial_{\eta_{k}} A_{0} \\
=-\sum \varphi_{i(j)}(y) \psi_{j(i, k)}(x) \partial_{\xi_{k}} A_{0}=-\sum \partial_{y_{j}}\left\{\psi_{j(k)}(\varphi(y))\right\} \partial_{\xi_{k}} A_{0} . \\
\text { Q.E.D. }
\end{array}
$$

\section{§. Space-like Change of Variables}

Let $\Gamma_{x}$ be the open connected component of $\left\{\xi ; A_{0}(x, \xi) \neq 0\right\}$ containing $\xi=(1,0, \cdots, 0)$. Let us say that $y=\psi(x)=\left(\psi_{0}(x), \cdots, \psi_{n}(x)\right)$ is 
a space-like change of variables if $\partial_{x} \psi_{0}(x) \in \Gamma_{x}$.

Lemma 8. 1. Let $y=\psi(x)$ be a space-like change of variables. If the Condition $(A)$ is satisfied by $A\left(x, D_{x}\right)$, then it is satisfied also by $\mathcal{A}\left(y, D_{y}\right)=A\left(\varphi(y),{ }^{t} \Psi(\varphi(y)) D_{y}\right)$.

Proof. We have from Lemma 7.2

$$
\mathcal{A}_{0}(y, \eta)=A_{0}\left(\varphi(y),{ }^{t} \Psi(\varphi(y)) \eta\right)=A_{0}\left(\varphi(y), \sum_{j=0}^{n} \partial_{x} \psi_{j}(\varphi(y)) \eta_{j}\right),
$$

therefore we have

$$
\mathcal{A}_{0}(y,(1,0, \cdots, 0))=A_{0}\left(\varphi(y),\left(\partial_{x} \psi_{0}\right)(\varphi(y))\right) \neq 0 .
$$

Moreover, since

$$
A_{0}(x, \xi) \neq 0 \quad \text { for } \quad \pm \operatorname{Im} \xi \in \Gamma_{x},
$$

we have

$$
\mathscr{A}_{0}(y, \eta) \neq 0 \quad \text { for } \quad \eta=\left(\omega, \eta^{\prime}\right), \quad \operatorname{Im} \omega \neq 0, \quad \eta^{\prime} \in R^{n} .
$$

Q.E.D.

Now, let us consider the decomposition of $A_{0}(x, \xi)$ at $X^{\prime}=X_{0}^{\prime}$ $=\left(x_{0}, \xi_{0}^{\prime}\right)$ :

$$
A_{0}(x, \xi)=\prod_{i=1}^{d} H_{i}(x, \xi) \prod_{i=d+1}^{m-d} h_{i}(x, \xi)
$$

then we have

Lemma 8. 2. Let $\zeta \in \Gamma_{x_{0}}$, then there exist $\varepsilon_{0}>0$ and $c>0$ such that

(i) $H_{i}\left(x_{0}, \xi_{0}+\varepsilon \zeta\right) \geq c \varepsilon^{2} \quad(i=1, \cdots, d)$,

(ii) $\quad h_{i}\left(x_{0}, \xi_{0}+\varepsilon \zeta\right) \geq c \varepsilon \quad(i=d+1, \cdots, m-d)$

for $0<\varepsilon<\varepsilon_{0}$, where $\hat{\xi}_{0}=\left(\alpha_{i}\left(X_{0}^{\prime}\right), \xi_{0}^{\prime}\right)$.

Proof. (i) Let

$$
H_{i}\left(x_{0}, \xi\right)=\left(\tau-\alpha_{i}\left(\xi^{\prime}\right)\right)^{2}-\beta_{i}\left(\xi^{\prime}\right)=\left(\tau-\tau_{+}\left(\xi^{\prime}\right)\right)\left(\tau-\tau_{-}\left(\xi^{\prime}\right)\right),
$$


where $\tau_{+}\left(\xi^{\prime}\right) \geq \tau_{-}\left(\xi^{\prime}\right)$, and let

$$
\Gamma_{x_{0}}^{(1)}=\left\{\zeta \in \Gamma_{x_{0}} ;|\zeta|<1\right\} .
$$

Then there exists $\varepsilon_{0}>0$ such that

$$
\xi_{0}+\varepsilon_{0} \Gamma_{x_{0}}^{(1)} \subset\left\{\tau>\tau_{+}\left(\xi^{\prime}\right)\right\},
$$

that is,

$$
\tau_{0}+\varepsilon \theta \geq \tau_{+}\left(\xi_{0}^{\prime}+\varepsilon \zeta^{\prime}\right) \text { for } 0<\varepsilon<\varepsilon_{0}, \zeta=\left(0, \zeta^{\prime}\right) \in \Gamma_{x_{0}}^{(1)},
$$

because $A_{0}\left(x_{0}, \xi\right)$ is hyperbolic with respect to each $\zeta \in \Gamma_{x_{0}}$. Let $\zeta=\left(\theta, \zeta^{\prime}\right)$

$\in \Gamma_{x_{0}}^{(1)}$ be fixed, then there exists $c>0$ such that

$$
\tau_{0}+\varepsilon \theta-\tau_{+}\left(\xi_{0}^{\prime}+\varepsilon \zeta^{\prime}\right) \geq c \varepsilon \text { for } 0<\varepsilon<\varepsilon_{0} .
$$

Because, choosing $\theta^{\prime}<\theta$ such that $\left(\theta^{\prime}, \zeta\right) \in \Gamma_{x_{0}}^{(1)}$, we have

$$
\tau_{0}+\varepsilon \theta^{\prime} \geq \tau_{+}\left(\xi_{0}^{\prime}+\varepsilon \zeta^{\prime}\right),
$$

that is

$$
\tau_{0}+\varepsilon \theta-\tau_{+}\left(\xi_{0}^{\prime}+\varepsilon \zeta^{\prime}\right)=\left\{\tau_{0}+\varepsilon \theta^{\prime}-\tau_{+}\left(\xi_{0}^{\prime}+\varepsilon \zeta^{\prime}\right)\right\}+\varepsilon\left(\theta-\theta^{\prime}\right) \geq \varepsilon\left(\theta-\theta^{\prime}\right) .
$$

Here we have

$$
\begin{aligned}
H_{i}\left(x_{0}, \xi_{0}+\varepsilon \zeta\right)= & \left(\tau_{0}+\varepsilon \theta-\tau_{+}\left(\xi_{0}^{\prime}+\varepsilon \zeta^{\prime}\right)\right)\left(\tau_{0}+\varepsilon 0-\tau_{-}\left(\xi_{0}^{\prime}+\varepsilon \zeta^{\prime}\right)\right) \\
& \geq\left(\tau_{0}+\varepsilon \theta-\tau_{+}\left(\xi_{0}^{\prime}+\varepsilon \zeta^{\prime}\right)\right)^{2} \geq \varepsilon^{2} c^{2} .
\end{aligned}
$$

(ii) Regarding $\alpha_{i}\left(\xi^{\prime}\right)$ as $\tau_{+}\left(\xi^{\prime}\right)$, we have in the same way

$$
h_{i}\left(x_{0}, \xi_{0}+\varepsilon \zeta\right)=\tau_{0}+\varepsilon \theta-\alpha_{i}\left(\xi_{0}^{\prime}+\varepsilon \zeta^{\prime}\right) \geq \varepsilon c \text { for } 0<\varepsilon<\varepsilon_{0} .
$$

Q.E.D.

Corollary. Let $\zeta=\left(\theta, \zeta^{\prime}\right) \in \Gamma_{x_{0}}$, then

(i) $\left\{\theta-\partial_{\xi}^{\prime} \alpha_{i}\left(X_{0}^{\prime}\right) \cdot \zeta^{\prime}\right\}^{2}-\frac{1}{2} \sum_{j, k=1}^{n} \partial_{\xi_{j}} \partial_{\xi_{k}} \beta_{i}\left(X_{0}^{\prime}\right) \zeta_{j} \zeta_{k}>0 \quad(i=1, \cdots, d)$,

(ii) $\quad \theta-\partial_{\xi}^{\prime} \alpha_{i}\left(X_{0}{ }^{\prime}\right) \cdot \zeta^{\prime}>0 \quad(i=d+1, \cdots, m-d)$.

Remark. $\theta-\partial_{\xi}^{\prime} \alpha_{i}\left(X_{0}^{\prime}\right) \cdot \zeta^{\prime}>0(i=1, \cdots, d)$ follow from (i), because $\beta_{i}\left(X^{\prime}\right) \geq 0, \beta_{i}\left(X_{0}^{\prime}\right)=0$.

Proof. (i) Let $X_{0}=\left(x_{0}, \alpha_{i}\left(x_{0}, \xi_{0}^{\prime}\right), \xi_{0}^{\prime}\right)$, then 


$$
H_{i}\left(X_{0}\right)=\partial_{\tau} H_{i}\left(X_{0}\right)=\partial_{\xi}^{\prime} H_{i}\left(X_{0}\right)=0,
$$

and

$$
\left\{\begin{array}{l}
\partial_{\tau}{ }^{2} H_{i}\left(X_{0}\right)=2, \quad \partial_{\tau} \partial_{\xi_{j}} H_{i}\left(X_{0}\right)=-2 \partial_{\xi_{j}} \alpha_{i}\left(X_{0}^{\prime}\right), \\
\partial_{\xi_{j}} \partial_{\xi_{k}} H_{i}\left(X_{0}\right)=2 \partial_{\xi_{j}} \alpha_{i}\left(X_{0}^{\prime}\right) \partial_{\xi_{k}} \alpha_{i}\left(X_{0}^{\prime}\right)-\partial_{\xi_{j}} \partial_{\xi_{k}} \beta_{i}\left(X_{0}^{\prime}\right) .
\end{array}\right.
$$

Then we have by Taylor's expansion

$$
\begin{gathered}
H_{i}\left(x_{0}, \xi_{0}+\varepsilon \zeta\right)=\frac{\varepsilon^{2}}{2}\left\{\partial_{\tau}^{2} H_{i}\left(X_{0}\right) \theta^{2}+2 \sum_{j=1}^{n} \partial_{\tau} \partial_{\xi_{j}} H_{i}\left(X_{0}\right) \theta \zeta_{j}\right. \\
\left.+\sum_{j, \prod_{k=1}^{n}} \partial_{\xi_{j}} \partial_{\xi_{k}} H_{i}\left(X_{0}\right) \zeta_{j} \zeta_{k}\right\}+0\left(\varepsilon^{3}\right) \\
=\varepsilon^{2}\left\{\left(\theta-\sum_{j=1}^{n} \partial_{\xi_{j}} \alpha_{i}\left(X_{0}^{\prime}\right) \zeta_{j}\right)^{2}-\frac{1}{2} \sum_{j, k=1}^{n} \partial_{\xi_{j}} \partial_{\xi_{k}} \beta_{i}\left(X_{0}^{\prime}\right) \zeta_{j} \zeta_{k}\right\}+0\left(\varepsilon^{3}\right) \\
\geq c \varepsilon^{2} \quad(c>0),
\end{gathered}
$$

which means

$$
\left(\theta-\sum_{j=1}^{n} \partial_{\xi_{j}} \alpha_{i}\left(X_{0}^{\prime}\right) \zeta_{j}\right)^{2}-\frac{1}{2} \sum_{j, k=1}^{n} \partial_{\xi_{j}} \partial_{\xi_{k}} \beta_{i}\left(X_{0}^{\prime}\right) \zeta_{j} \zeta_{k}>0
$$

(ii) Since

$$
h_{i}\left(X_{0}\right)=0, \partial_{\tau} h_{i}\left(X_{0}\right)=1, \partial_{\xi_{j}} h_{i}\left(X_{0}\right)=-\partial_{\xi_{j}} \alpha_{i}\left(X_{0}^{\prime}\right),
$$

we have

$$
h_{i}\left(x_{0}, \xi_{0}+\varepsilon \zeta\right)=\varepsilon\left\{\theta-\sum_{j=1}^{n} \partial_{\xi_{j}} \alpha_{i}\left(X_{0}^{\prime}\right) \zeta_{j}\right\}+0\left(\varepsilon^{2}\right) \geq c \varepsilon \quad(c>0),
$$

which means

$$
\theta-\sum_{j=1}^{n} \partial_{\xi_{j}} \alpha_{i}\left(X_{0}^{\prime}\right) \zeta_{j}>0
$$

Now denoting

$$
Y=(y, \eta)=\left(y, \omega, \eta^{\prime}\right), \quad Y^{\prime}=\left(y, \eta^{\prime}\right),
$$

we consider the decomposition of $\mathcal{A}_{0}(Y)$ at $Y^{\prime}=Y_{0}^{\prime}=\left(y_{0}, \eta_{0}^{\prime}\right)$. Let $\omega_{0}$ be a root of $\mathscr{A}_{0}\left(\omega, Y^{\prime}\right)=0$, and let $Y_{0}=\left(\omega_{0}, Y_{0}^{\prime}\right)$, then we have $A_{0}\left(X_{0}\right)$ $=0$, where

$$
X_{0}=\left(x_{0}, \xi_{0}\right)=\left(\varphi\left(y_{0}\right),{ }^{t} \Psi\left(\varphi\left(y_{0}\right)\right) \eta_{0}\right)
$$


Here, we consider the decomposition of $A_{0}(X)$ at $X^{\prime}=X_{0}^{\prime}=\left(x_{0}, \xi_{0}^{\prime}\right)$ :

$$
A_{0}(X)=\prod_{i=1}^{d} H_{i}(X) \prod_{i=d+1}^{m-d} h_{i}(X),
$$

then there exists unique number $i(\leq d)$ such that $H_{i}\left(X_{0}\right)=0$ or $i(>d)$ such that $h_{i}\left(X_{0}\right)=0$. First, we consider the case of $i \leq d$, then we have

$$
\begin{aligned}
\left.\partial_{\omega}\left\{H_{i}\left(x_{0}, \partial_{x} \psi_{0}\left(x_{0}\right) \omega+\sum_{j=1}^{n} \partial_{x} \psi_{j}\left(x_{0}\right) \eta_{j}^{0}\right)\right\}\right|_{\omega=\omega_{0}} \\
=\left(\partial_{\tau} H_{i}\right)\left(X_{0}\right)\left(\partial_{t} \psi_{0}\right)\left(x_{0}\right)+\sum_{j=1}^{n}\left(\partial_{\xi_{j}} H_{i}\right)\left(X_{0}\right)\left(\partial_{x_{j}} \psi_{0}\right)\left(x_{0}\right)=0, \\
\left.\partial_{\omega}{ }^{2}\left\{H_{i}\left(x_{0}, \partial_{x} \psi_{0}\left(x_{0}\right) \omega+\sum_{j=1}^{n} \partial_{x} \psi_{j}\left(x_{0}\right) \eta_{j}{ }^{0}\right)\right\}\right|_{\omega=\omega_{0}} \\
=\left(\partial_{\tau}{ }^{2} H_{i}\right)\left(X_{0}\right)\left\{\left(\partial_{t} \psi_{0}\right)\left(x_{0}\right)\right\}^{2}+2 \sum_{j=1}^{n}\left(\partial_{\tau} \partial_{\xi_{j}} H_{i}\right)\left(X_{0}\right)\left(\partial_{t} \psi_{0}\right)\left(x_{0}\right) \\
\quad \times\left(\partial_{x_{j}} \psi_{0}\right)\left(x_{0}\right)+\sum_{j, k=1}^{n}\left(\partial_{\xi_{j}} \partial_{\xi_{k}} H_{i}\right)\left(X_{0}\right)\left(\partial_{x_{x}} \psi_{0}\right)\left(x_{0}\right)\left(\partial_{x_{k}} \psi_{0}\right)\left(x_{0}\right) \\
=2\left[\left\{\left(\partial_{t} \psi_{0}\right)\left(x_{0}\right)-\sum_{j=1}^{n}\left(\partial_{\xi_{j}} \alpha_{i}\right)\left(X_{0}^{\prime}\right)\left(\partial_{x_{j}} \psi_{0}\right)\left(x_{0}\right)\right\}^{2}\right. \\
\left.\quad-\frac{1}{2} \sum_{j, k=1}^{n}\left(\partial_{\xi_{j}} \partial_{\xi_{k}} \beta_{i}\right)\left(X_{0}^{\prime}\right)\left(\partial_{x_{j}} \psi_{0}\right)\left(x_{0}\right)\left(\partial_{x_{k}} \psi_{0}\right)\left(x_{0}\right)\right]>0 .
\end{aligned}
$$

Then, using Weierstrass' preparation theorem, we have

$$
\begin{aligned}
& H_{i}\left(\varphi(y),{ }^{t} \Psi(\varphi(y)) \eta\right)=H_{i}\left(x, \partial_{x} \psi_{0}(x) \omega+\sum_{j=1}^{n} \partial_{x} \psi_{j}(x) \eta_{j}\right) \\
& \quad=\left\{\left(\omega-\tilde{\alpha}\left(y, \eta^{\prime}\right)\right)^{2}-\tilde{\beta}\left(y, \eta^{\prime}\right)\right\} f\left(y, \omega, \eta^{\prime}\right) \\
& \quad=\left\{\left(\omega-\tilde{\alpha}\left(Y^{\prime}\right)\right)^{2}-\tilde{\beta}\left(Y^{\prime}\right)\right\} f(Y),
\end{aligned}
$$

where

$$
\tilde{\alpha}\left(Y_{0}^{\prime}\right)=\omega_{0}, \tilde{\beta}\left(Y_{0}^{\prime}\right)=0, f\left(Y_{0}\right)>0 .
$$

In case of $i>d$, we have by the similar reasoning

$$
h_{i}\left(\varphi(y),{ }^{t} \Psi(\varphi(y)) \eta\right)=\left(\omega-\tilde{\alpha}\left(Y^{\prime}\right)\right) f(Y),
$$

where

$$
\widetilde{\alpha}\left(Y_{0}^{\prime}\right)=\omega_{0}, \quad f\left(Y_{0}\right)>0 \text { 。 }
$$

Hence roots of $\mathscr{A}_{0}\left(\omega, Y_{0}^{\prime}\right)=0$ are double at most, and therefore we 
have its smooth decomposition at $Y^{\prime}=Y_{0}^{\prime}$ :

$$
\mathcal{A}_{0}(Y)=c(y) \prod_{j=1}^{\grave{o}} \widetilde{H}_{j}(Y) \prod_{j=\hat{\delta}+1}^{m-\hat{o}} \tilde{h}_{j}(Y)
$$

where

$$
\left\{\begin{array}{l}
\tilde{H}_{j}(Y)=\left(\omega-\tilde{\alpha}_{j}\left(Y^{\prime}\right)\right)^{2}-\tilde{\beta}_{j}\left(Y^{\prime}\right) \quad(j=1, \cdots, \delta), \\
\tilde{h}_{j}(Y)=\omega-\tilde{\alpha}_{j}\left(Y^{\prime}\right) \quad(j=\hat{0}+1, \cdots, m-\delta) .
\end{array}\right.
$$

Let $Y_{0}=\left(\widetilde{\alpha}_{j}\left(Y_{0}^{\prime}\right), Y_{0}^{\prime}\right)$, then it corresponds

$$
X_{0}=X\left(Y_{0}\right)=\left(\varphi\left(y_{0}\right),{ }^{t} \Psi\left(\varphi\left(y_{0}\right)\right) \eta_{0}\right)
$$

and let

$$
A_{0}(X)=\prod_{i=1}^{d} H_{i}(X) \prod_{i=d+1}^{m-d} h_{i}(X)
$$

be the decomposition of $A_{0}(X)$ at $X^{\prime}=X_{0}^{\prime}$, then we have

Lemma 8. 3. If $j \leq \delta$, then there exists $i(\leq d)$ such that

$$
\begin{aligned}
& H_{i}(X(Y))=\tilde{H}_{j}(Y) f_{j}(Y), \quad f_{j}\left(Y_{0}\right)>0, \\
& h_{i}(X(Y))=\left(\omega-\tilde{\widetilde{\alpha}}_{j}\left(Y^{\prime}\right)\right) g_{j}(Y), g_{j}\left(Y_{0}\right) \neq 0,
\end{aligned}
$$

and if $j>\delta$, then there exists $i(>d)$ such that

$$
h_{i}(X(Y))=\tilde{h}_{j}(Y) f_{j}(Y), \quad f_{j}\left(Y_{0}\right)>0,
$$

where $f_{j}(Y)$ and $g_{j}(Y)$ are smooth in a neighbourhood of $Y=Y_{0}$.

\section{$\S$ 9. Space-like Change of Variables and Condition (B)}

Now we consider whether condition (B) is satisfied or not for $\mathcal{A}$, given by space-like change of variables of $A$. Let

$$
\begin{aligned}
\mathcal{A}_{0}(Y) & =c \prod_{j=1}^{\delta} \tilde{H}_{j}(Y) \prod_{j=\hat{o}+1}^{m-\hat{o}} \tilde{h}_{j}(Y) \\
& =c \prod_{j=1}^{\delta}\left\{\left(\omega-\widetilde{\alpha}_{j}\left(Y^{\prime}\right)\right)^{2}-\tilde{\beta}_{j}\left(Y^{\prime}\right)\right\} \prod_{j=\delta+1}^{m-\delta}\left(\omega-\widetilde{\alpha}_{j}\left(Y^{\prime}\right)\right) .
\end{aligned}
$$

be the decomposition of $\mathscr{A}_{0}(Y)$ at $Y^{\prime}=Y_{0}^{\prime}$. Let $j \leq \delta$, then there exists the decomposition of $A_{0}(X)$ at $X^{\prime}=X_{0}^{\prime}=X^{\prime}\left(Y_{0}\right)=X^{\prime}\left(\alpha_{j}\left(Y_{0}^{\prime}\right), Y_{0}^{\prime}\right)$ : 


$$
\begin{aligned}
A_{0}(X) & =c \prod_{i=1}^{d} H_{i}(X) \prod_{i=d+1}^{m-d} h_{i}(X) \\
& =c \prod_{i=1}^{d}\left\{\left(\tau-\alpha_{i}\left(X^{\prime}\right)\right)^{2}-\beta_{i}\left(X^{\prime}\right)\right\} \prod_{i=d+1}^{m-d}\left(\tau-\alpha_{i}\left(X^{\prime}\right)\right)
\end{aligned}
$$

where there exists $i(\leq d)$ such that

$$
H_{i}(X)=\widetilde{H}_{j}(Y) f_{j}(Y), f_{j}(Y)>0,
$$

that is,

$$
\left(\tau-\alpha_{i}\left(X^{\prime}\right)\right)^{2}-\beta_{i}\left(X^{\prime}\right)=\left\{\left(\omega-\tilde{\alpha}_{j}\left(Y^{\prime}\right)\right)^{2}-\tilde{\beta}_{j}\left(Y^{\prime}\right)\right\} f_{j}(Y) .
$$

Moreover, we have

$$
\tau-\alpha_{i}\left(X^{\prime}\right)=\left(\omega-\tilde{\tilde{\alpha}}_{j}\left(Y^{\prime}\right)\right) g_{j}(Y), g_{j}(Y) \neq 0,
$$

then we have

$$
\begin{aligned}
& \left(\omega-\tilde{\tilde{\alpha}}_{j}\left(Y^{\prime}\right)\right)^{2} g_{j}(Y)^{2}-\beta_{i}\left(X^{\prime}(Y)\right) \\
& \quad=\left\{\left(\omega-\widetilde{\alpha}_{j}\left(Y^{\prime}\right)\right)^{2}-\hat{\beta}_{j}\left(Y^{\prime}\right)\right\} f_{j}(Y) .
\end{aligned}
$$

Let $\omega=\tilde{\tilde{\alpha}}_{j}\left(Y^{\prime}\right)$, then we have

$$
\begin{gathered}
\tilde{\beta}_{j}\left(Y^{\prime}\right)=f_{j}\left(\tilde{\tilde{\alpha}}_{j}\left(Y^{\prime}\right), Y^{\prime}\right)^{-1} \beta_{i}\left(X^{\prime}\left(\tilde{\tilde{\alpha}}_{j}\left(Y^{\prime}\right), Y^{\prime}\right)\right. \\
+\left(\tilde{\tilde{\alpha}}_{j}\left(Y^{\prime}\right)-\widetilde{\alpha}_{j}\left(Y^{\prime \prime}\right)\right)^{2}
\end{gathered}
$$

Let

$$
\beta_{i}\left(X^{\prime}\right)=\sum_{k=1}^{N_{i}} b_{k}^{(i)}\left(X^{\prime}\right)^{2}
$$

then we have

$$
\tilde{\beta}_{j}\left(Y^{\prime}\right)=\sum_{k=1}^{\tilde{N}_{j}} b_{k}^{(j)}\left(Y^{\prime}\right)^{2} \quad\left(\widetilde{N}_{j}=N_{i}+1\right)
$$

where

$$
\begin{aligned}
& \tilde{b}_{k}^{(j)}\left(Y^{\prime}\right)=f_{j}\left(\tilde{\tilde{\alpha}}_{j}\left(Y^{\prime}\right), Y^{\prime}\right)^{-1 / 2} b_{k}^{(i)}\left(X^{\prime}\left(\tilde{\tilde{\alpha}}_{j}\left(Y^{\prime}\right), Y^{\prime}\right)\right) \quad\left(k=1, \cdots, N_{i}\right), \\
& b_{N_{i}+1}^{(j)}\left(Y^{\prime}\right)=\tilde{\tilde{\alpha}}_{j}\left(Y^{\prime}\right)-\tilde{\alpha}_{j}\left(Y^{\prime}\right) .
\end{aligned}
$$

Hence

Lemma 9.1. Let (B.1) be satisfied for 1 , then it is also satisfied for $A$. 
Next, differentiate $(*)$ with respect to $\omega$ and let $\omega=\tilde{\tilde{\alpha}}_{j}\left(Y^{\prime}\right)$, then we have

$$
\begin{aligned}
& -\left(\partial_{\omega} \beta_{i}\right)\left(X^{\prime}\left(\tilde{\tilde{\alpha}}_{j}\left(Y^{\prime}\right), Y^{\prime}\right)\right)=2\left(\tilde{\tilde{\alpha}}_{j}\left(Y^{\prime}\right)-\tilde{\alpha}_{j}\left(Y^{\prime}\right)\right) f_{j}\left(\tilde{\alpha}_{j}\left(Y^{\prime}\right), Y^{\prime}\right) \\
& \quad+\left\{\left(\tilde{\tilde{\alpha}}_{j}\left(Y^{\prime}\right)-\tilde{\alpha}_{j}\left(Y^{\prime}\right)\right)^{2}-\tilde{\beta}_{j}\left(Y^{\prime}\right)\right\}\left(\partial_{\omega} f_{j}\right)\left(\tilde{\tilde{\alpha}}_{j}\left(Y^{\prime}\right), Y^{\prime}\right) \\
& =2\left(\tilde{\tilde{\alpha}}_{j}\left(Y^{\prime}\right)-\tilde{\alpha}_{j}\left(Y^{\prime}\right)\right) f_{j}\left(\tilde{\tilde{\alpha}}_{j}\left(Y^{\prime}\right), Y^{\prime}\right) \\
& -\beta_{i}\left(X^{\prime}\left(\tilde{\tilde{\alpha}}_{j}\left(Y^{\prime}\right), Y^{\prime}\right)\right) f_{j}\left(\tilde{\tilde{\alpha}}_{j}\left(Y^{\prime}\right), Y^{\prime}\right)^{-1}\left(\partial_{\omega} f_{j}\right)\left(\tilde{\tilde{\alpha}}_{j}\left(Y^{\prime}\right), Y^{\prime}\right),
\end{aligned}
$$

that is,

$$
\begin{gathered}
\tilde{\tilde{\alpha}}_{j}\left(Y^{\prime}\right)-\tilde{\alpha}_{j}\left(Y^{\prime}\right)=\frac{1}{2} f_{j}\left(\tilde{\tilde{\alpha}}_{j}\left(Y^{\prime}\right), Y^{\prime}\right)^{-1}\left\{-\left(\partial_{\omega} \beta_{i}\right)\left(X^{\prime}\left(\tilde{\tilde{\alpha}}_{j}\left(Y^{\prime}\right), Y^{\prime}\right)\right)\right. \\
\left.\left.+\beta_{i}\left(X^{\prime}\left(\tilde{\tilde{\alpha}}_{j}\left(Y^{\prime}\right), Y^{\prime}\right)\right) f_{j}\left(\tilde{\tilde{\alpha}}_{j}\left(Y^{\prime}\right), Y^{\prime}\right)\right)^{-1}\left(\partial_{\omega} f_{j}\right)\left(\tilde{\tilde{\alpha}}_{j}(Y), Y\right)\right) .
\end{gathered}
$$

Hence

Lemma 9. 2. Let (B.1) be satisfied for $A$, then

$$
\tilde{\tilde{\alpha}}_{j}\left(Y^{\prime}\right)-\tilde{\alpha}_{j}\left(Y^{\prime}\right) \equiv 0 \bmod \left(\tilde{b}_{1}^{(j)}\left(Y^{\prime}\right), \cdots, \tilde{b}_{N_{j-1}}^{(j)}\left(Y^{\prime}\right)\right)
$$

Corollary. Let (B.1) and (B.2) be satisfied for $A$, then (B.2) is satisfied also for $\mathcal{A}$.

Proof. We have from Lemma 7.2 and Lemma 9.2

$$
\begin{aligned}
L_{\mathfrak{A}}\left(\tilde{\alpha}_{j}\left(Y^{\prime}\right), Y^{\prime}\right) \equiv L_{\mathfrak{A}}\left(\tilde{\tilde{\alpha}}_{j}\left(Y^{\prime}\right), Y^{\prime}\right) \equiv L\left(\alpha_{i}\left(X^{\prime}\right), X^{\prime}\right) \\
\\
\bmod \tilde{\boldsymbol{b}}^{(j)}\left(Y^{\prime}\right) .
\end{aligned}
$$

Finally, we consider of Conditions (B. 3) and (B. 4). Condition (B. 3) for $A$ is

$$
\left\{h_{i}(X), b_{k}^{(i)}\left(X^{\prime}\right)\right\} \equiv 0 \bmod \boldsymbol{b}^{(i)}\left(X^{\prime}\right),
$$

which is equivalent to

$$
\left\{\left(\omega-\tilde{\tilde{\alpha}}_{j}\left(Y^{\prime}\right)\right) g_{j}(Y), b_{k}^{(i)}\left(X^{\prime}(Y)\right)\right\} \equiv 0 \bmod \boldsymbol{b}^{(i)}\left(X^{\prime}(Y)\right)
$$

from the corollary of Lemma 7.1. Let $\omega=\tilde{\tilde{\alpha}}_{j}\left(Y^{\prime}\right)$ in the latter equality, then we have

$$
\left\{\omega-\tilde{\tilde{\alpha}}_{j}\left(Y^{\prime}\right), \tilde{b}_{k}^{(j)}\left(Y^{\prime}\right)\right\} \equiv 0 \bmod \tilde{\boldsymbol{b}}^{(j)}\left(Y^{\prime}\right) .
$$


In the same way, Condition (B. 4) is

$$
\left\{b_{k}^{(i)}\left(X^{\prime}\right), b_{l}^{(i)}\left(X^{\prime}\right)\right\} \equiv 0 \bmod b^{(i)}\left(X^{\prime}\right),
$$

which is equivalent to

$$
\left\{b_{k}^{(i)}\left(X^{\prime}(Y)\right), b_{l}^{(i)}\left(X^{\prime}(Y)\right)\right\} \equiv 0 \bmod b^{(i)}\left(X^{\prime}(Y)\right) .
$$

Let $\omega=\widetilde{\tilde{\alpha}}_{j}\left(Y^{\prime}\right)$, then we have

$$
\begin{gathered}
\left\{\tilde{b}_{k}^{(j)}\left(Y^{\prime}\right)+\partial_{\omega} b_{k}^{(i)}\left(X^{\prime}\left(\tilde{\tilde{\alpha}}_{j}\left(Y^{\prime}\right), Y^{\prime}\right)\right)\left(\omega-\tilde{\tilde{\alpha}}_{j}\left(Y^{\prime}\right)\right),\right. \\
\left.\tilde{b}_{l}^{(j)}\left(Y^{\prime}\right)+\partial_{\omega} b_{l}^{(i)}\left(X^{\prime}\left(\tilde{\tilde{\alpha}}_{j}\left(Y^{\prime}\right), Y^{\prime}\right)\right)\left(\omega-\tilde{\tilde{\alpha}}_{j}\left(Y^{\prime}\right)\right)\right\}_{\omega=\tilde{\alpha}_{j}\left(Y^{\prime}\right)} \equiv 0 \\
\bmod \tilde{b}^{(j)}\left(Y^{\prime}\right) .
\end{gathered}
$$

Since

$$
\left\{\omega-\tilde{\tilde{\alpha}}_{j}\left(Y^{\prime}\right), \tilde{b}_{k}^{(j)}\left(Y^{\prime}\right)\right\} \equiv 0 \bmod \tilde{b}^{(j)}\left(Y^{\prime}\right)
$$

we have

$$
\left\{\tilde{b}_{k}^{(j)}\left(Y^{\prime}\right), \tilde{b}_{l}^{(j)}\left(Y^{\prime}\right)\right\} \equiv 0 \bmod \tilde{b}^{(j)}\left(Y^{\prime}\right)
$$

On the other hands, we have from Lemma 9.2

$$
\tilde{\alpha}_{j}\left(Y^{\prime}\right)-\tilde{\alpha}_{j}\left(Y^{\prime}\right)=\sum_{l} c_{l}\left(Y^{\prime}\right) \tilde{b}_{l}^{(j)}\left(Y^{\prime}\right)
$$

therefore

$$
\begin{aligned}
&\left\{\tilde{\tilde{\alpha}}_{j}\left(Y^{\prime}\right)-\widetilde{\alpha}_{j}\left(Y^{\prime}\right), \tilde{b}_{k}^{(j)}\left(Y^{\prime}\right)\right\} \equiv \sum_{i} c_{l}\left(Y^{\prime}\right)\left\{\tilde{b}_{l}^{(j)}\left(Y^{\prime}\right), \tilde{b}_{k}^{(j)}\left(Y^{\prime}\right)\right\} \equiv 0 \\
& \bmod \tilde{b}^{(j)}\left(Y^{\prime}\right)
\end{aligned}
$$

Moreover, we have

$$
\begin{aligned}
& \left\{\omega-\widetilde{\alpha}_{j}\left(Y^{\prime}\right), \tilde{b}_{k}^{(j)}\left(Y^{\prime}\right)\right\}=\left\{\omega-\tilde{\tilde{\alpha}}_{j}\left(Y^{\prime}\right), \tilde{b}_{k}^{(j)}\left(Y^{\prime}\right)\right\} \\
& \quad+\left\{\tilde{\tilde{\alpha}}_{j}\left(Y^{\prime}\right)-\widetilde{\alpha}_{j}\left(Y^{\prime}\right), \tilde{b}_{k}^{(j)}\left(Y^{\prime}\right)\right\} \equiv 0 \bmod \tilde{b}^{(j)}\left(Y^{\prime}\right) .
\end{aligned}
$$

Here we have

Lemma 9.3. Let us assume conditions (B.1), (B.3), (B.4) for $A$, then we have (B.3), (B.4) for $\mathcal{A}$,

Proposition 9. 4. Let us assume that Conditions $(A)$ and $(B)$ are satisfied by $A$, then they are satisfied also by $\mathcal{A}$ which is given by a space-like change of variables of $A$. 


\section{$\S 10$. Finiteness of Propagation Speed}

Let us denote $\Gamma_{x}$ be the connected component of

$$
\left\{\xi \in \boldsymbol{R}^{n+1} ; A_{0}(x, \xi) \neq 0\right\},
$$

containing $\xi=(1,0, \cdots, 0) . \quad \Gamma_{x}$ is an open convex cone. Let

$$
\begin{gathered}
A_{0}(x, \xi)=\prod_{j=1}^{m}\left(\tau-\tau_{j}\left(x, \xi^{\prime}\right)\right), \xi=\left(\tau, \xi^{\prime}\right), \\
\tau\left(x, \xi^{\prime}\right)=\max _{j} \tau_{j}\left(x, \xi^{\prime}\right),
\end{gathered}
$$

then

$$
\Gamma_{x}=\left\{\xi \in \mathbb{R}^{n+1} ; \tau>\tau\left(x, \xi^{\prime}\right)\right\}
$$

Moreover, we denote

$$
\Gamma=\text { interior of } \bigcap_{x} \Gamma_{x},
$$

then $\Gamma$ is also an open convex cone and

$$
\Gamma=\left\{\xi=\left(\tau, \xi^{\prime}\right) \in R^{n+1} ; \tau>\tau\left(\xi^{\prime}\right)\right\}, \quad \tau\left(\xi^{\prime}\right)=\sup _{x} \tau\left(x, \xi^{\prime}\right),
$$

where $\tau\left(\xi^{\prime}\right)$ is continuous in $\boldsymbol{R}^{n}-\{0\}$, because of the convexity of $\Gamma$.

Next, we consider the dual cone $\Gamma^{\prime}$ of $\Gamma$ :

$$
\Gamma^{\prime}=\left\{x \in \boldsymbol{R}^{n+1} ; x \cdot \xi \geq 0, \forall \xi \in \Gamma\right\} .
$$

Let us fix $a=\left(1, a^{\prime}\right) \in \dot{\Gamma}^{\prime}$, then we have a change of variables

$$
\left(\eta_{0}, \eta^{\prime}\right)=\left(a \cdot \xi, \xi^{\prime}\right) \quad \text { i.e. } \quad\left(\xi_{0}, \xi^{\prime}\right)=\left(\eta_{0}-a^{\prime} \cdot \eta^{\prime}, \eta^{\prime}\right) .
$$

If $\tau\left(\xi^{\prime}\right)$ is smooth, then $S=\partial \Gamma \cap\{a \cdot \xi=1\}$ is represented by

$$
S=\left\{\xi=\left(\eta_{0}-a^{\prime} \cdot \eta^{\prime}, \eta^{\prime}\right) ; \eta_{0}=1,1-a^{\prime} \cdot \eta^{\prime}=\tau\left(\eta^{\prime}\right)\right\},
$$

and $S^{\prime}=\partial \Gamma^{\prime} \cap\left\{x_{0}=1\right\}$ is represented by

$$
S^{\prime}=\left\{x=\left(x_{0}, x^{\prime}\right) ; x_{0}=1, x^{\prime}=-\partial_{\xi}^{\prime} \tau\left(\xi^{\prime}\right), \xi^{\prime} \in \boldsymbol{R}^{n}-\{0\}\right\} .
$$

Moreover if

$$
\operatorname{rank}\left(\partial_{\xi_{i}} \partial_{\xi_{j}} \tau\left(\xi^{\prime}\right)\right)_{i, j=1, \ldots, n}=n-1
$$

for $\xi^{\prime} \in R^{n}-\{0\}$, then $S^{\prime}$ becomes a smooth manifold of dimension $n-1$. Then there exists a smooth function $f\left(x^{\prime}\right)$ such that 


$$
\left\{\begin{array}{l}
S^{\prime}=\left\{x^{\prime} ; f\left(x^{\prime}\right)=0\right\}, \\
\text { interior domain of } S^{\prime}=\left\{x^{\prime} ; f\left(x^{\prime}\right)>0\right\},
\end{array}\right.
$$

that is,

$$
\Gamma^{\prime}=\left\{x=\left(x_{0}, x^{\prime}\right) ; x_{0}>0, f\left(\frac{x^{\prime}}{x_{0}}\right)>0\right\}
$$

In general, we shall see that $\Gamma$ can be approximated from the inside by such cones stated above, that is,

Lemma 10.1. There exist smooth open convex cones $\left\{\Gamma_{k}\right\}_{k=1,2, \ldots}$ such that

$$
\bar{\Gamma}_{k} \subset \Gamma_{k+1}, \bigcup_{k=1}^{\infty} \Gamma_{k}=\Gamma
$$

and

$$
\operatorname{rank}\left(\partial_{\xi_{i}} \partial_{\xi_{j}} \tau_{k}\left(\xi^{\prime}\right)\right)_{i, j=1, \cdots, n}=n-1
$$

where

$$
\Gamma_{k}=\left\{\xi=\left(\tau, \xi^{\prime}\right) ; \tau>\tau_{k}\left(\xi^{\prime}\right)\right\}
$$

Proof. Since $\Omega=\Gamma \cap\{a \cdot \xi=1\}$ is convex, there exist smooth and strictly convex domains $\left\{\Omega_{k}\right\}_{k=1,2, \ldots}$ such that

$$
\bar{\Omega}_{k} \subset \Omega_{k+1}, \bigcup_{k=1}^{\infty} \Omega_{k}=\Omega .
$$

Let

$$
\Gamma_{k}=\text { convex hull of } \Omega_{k} \text { and }\{0\},
$$

then we have the desired properties.

Q.E.D.

Corollary. $\Gamma_{k}^{\prime}$ and $\Gamma^{\prime}$ are represented by

$$
\begin{aligned}
& \Gamma_{k}^{\prime}=\left\{x=\left(x_{0}, x^{\prime}\right) ; x_{0}>0, f_{k}\left(\frac{x^{\prime}}{x_{0}}\right)>0\right\}, \\
& \Gamma^{\prime}=\bigcap_{k=1}^{\infty} \Gamma_{k}^{\prime}=\left\{x=\left(x_{0}, x^{\prime}\right) ; x_{0}>0, f\left(\frac{x^{\prime}}{x_{0}}\right)>0\right\},
\end{aligned}
$$

where 
516

REIKO SAKAMOTO

$$
f\left(x^{\prime}\right)=\inf _{k} f_{k}\left(x^{\prime}\right), \quad \partial_{x}\left\{f_{k}\left(\frac{x^{\prime}}{x_{0}}\right)\right\} \in \bar{\Gamma}_{k} \subset \Gamma \text {. }
$$

Lemma 10.2. Let $T>0$, then there exist smooth approximaling functions $\left\{\varphi_{j}(x)\right\}_{j=1,2 \ldots}$ such that $\partial_{x} \varphi_{j}(x) \in \Gamma$ and

$$
D_{1} \subset D_{2} \subset \cdots, \bigcup_{j=1}^{\infty} D_{j}=\stackrel{\circ}{\Gamma}^{\prime} \cap\left\{0<x_{0}<T\right\},
$$

where

$$
D_{j}=\left\{x=\left(x_{0}, x^{\prime}\right) ; \phi_{j}(x)>0,0<x_{0}<T\right\}
$$

Proof In the corollary of Lemma 10.1, we have

$$
\Gamma_{k}^{\prime}=\left\{x=\left(x_{0}, x^{\prime}\right) ; x_{0}>0, f_{k}\left(\frac{x^{\prime}}{x_{0}}\right)>0\right\}
$$

where

$$
a \cdot \partial_{x} f_{k}\left(\frac{x^{\prime}}{x_{0}}\right)=\left(\partial_{x_{0}} f_{k}+a^{\prime} \cdot \partial_{x}^{\prime} f_{k}\right)\left(\frac{x^{\prime}}{x_{0}}\right)>0
$$

Let us consider the change of variable: $\left(x_{0}, x^{\prime}\right)=\left(y_{0}, y^{\prime}+a^{\prime} \cdot y_{0}\right)$ i.e. $\left(y_{0}, y^{\prime}\right)=\left(x_{0}, x^{\prime}-a^{\prime} \cdot x_{0}\right)$. Then we have $\partial_{y_{0}}=\partial_{x_{0}}+a^{\prime} \cdot \partial_{x}^{\prime}$, and $\partial_{y}^{\prime}=\partial_{x}^{\prime}$. Hence, $f_{k}\left(\frac{x^{\prime}}{x_{0}}\right)=0$ can be solved with respect to $y_{0}$ :

$$
y_{0}=\varphi_{k}\left(y^{\prime}\right)
$$

that is,

$$
x_{0}=\varphi_{k}\left(x^{\prime}-a^{\prime} \cdot x_{0}\right) .
$$

Since $\partial_{x}\left\{f_{k}\left(\frac{x^{\prime}}{x_{0}}\right)\right\} \in \bar{\Gamma}_{k}$, we have

$$
\begin{aligned}
& \left(\left(\partial_{y_{0}}-a^{\prime} \cdot \partial_{y}^{\prime}\right)\left(y_{0}-\varphi_{k}\left(y^{\prime}\right)\right), \partial_{y}^{\prime}\left(y_{0}-\varphi_{k}\left(y^{\prime}\right)\right)\right) \\
& \quad=\left(1+a^{\prime} \cdot \partial_{y}^{\prime} \varphi_{k}\left(y^{\prime}\right),-\partial_{y}^{\prime} \varphi_{k}\left(y^{\prime}\right)\right) \in \bar{\Gamma}_{k} \subset \Gamma .
\end{aligned}
$$

Moreover

$$
\varphi\left(y^{\prime}\right)=\sup _{k} \varphi_{k}\left(y^{\prime}\right), \quad 0<\rho_{k}=\sup _{\varphi\left(y^{\prime}\right)<T}\left\{\varphi\left(y^{\prime}\right)-\varphi_{k}\left(y^{\prime}\right)\right\} \underset{k \rightarrow+\infty}{\longrightarrow} 0,
$$

where we may assume

$$
\sum_{k=1}^{\infty} \rho_{k}<+\infty
$$


Let

$$
\psi_{j}\left(y^{\prime}\right)=\varphi_{j}\left(y^{\prime}\right)+\sum_{k=j}^{\infty} \rho_{k}
$$

then we have

$$
\psi_{1}\left(y^{\prime}\right)>\psi_{2}\left(y^{\prime}\right)>\cdots \rightarrow \varphi\left(y^{\prime}\right) \text { in } \varphi\left(y^{\prime}\right)<T .
$$

Now, we operate Friedrichs' mollifier $\rho_{\varepsilon} *$ on each $\psi_{j}\left(y^{\prime}\right)$, then there exist $\varepsilon_{j}>0$ such that

$$
\widetilde{\psi}_{1}\left(y^{\prime}\right)>\widetilde{\psi}_{2}\left(y^{\prime}\right)>\cdots \rightarrow \varphi\left(y^{\prime}\right) \text { in } \varphi\left(y^{\prime}\right)<T,
$$

where

$$
\widetilde{\psi}_{j}\left(y^{\prime}\right)=\rho_{\varepsilon_{j}}\left(y^{\prime}\right) * \psi_{j}\left(y^{\prime}\right)
$$

Let

$$
\phi_{j}(x)=x_{0}-\widetilde{\psi}_{j}\left(x^{\prime}-a^{\prime} x_{0}\right), \quad D_{j}=\left\{\phi_{j}(x)>0\right\},
$$

then

$$
\begin{aligned}
\partial_{x} \phi_{j}(x) & =\left(1+a^{\prime} \cdot\left(\partial_{y}^{\prime} \widetilde{\psi}_{j}\right)\left(x^{\prime}-a^{\prime} x_{0}\right),-\partial_{y}^{\prime} \widetilde{\psi}_{j}\left(x^{\prime}-a^{\prime} x_{0}\right)\right) \\
& =\rho_{\varepsilon_{j}} *\left(1+a^{\prime} \cdot \partial_{y}^{\prime} \psi_{j},-\partial_{y}^{\prime} \psi_{j}\right) \in \Gamma .
\end{aligned}
$$

Using the sweeping out method, we have

Theorem III. Let us assume Conditions (A) and (B). Let $a=\left(a_{0}, a^{\prime}\right) \in \mathbb{R}^{n+1}\left(a_{0}>0\right)$ and $u$ satisfy

$$
\left\{\begin{array}{l}
A u=0 \text { in }\left\{a-\Gamma^{\prime}\right\} \cap\left\{x_{0}>0\right\}, \\
D_{t}^{j} u=0(j=0, \cdots, m-1) \text { on }\left\{a-\Gamma^{\prime}\right\} \cap\left\{x_{0}=0\right\},
\end{array}\right.
$$

then

$$
u=0 \quad \text { in }\left\{a-\Gamma^{\prime}\right\} \cap\left\{x_{0}>0\right\} \text {. }
$$

Corollary. Theorems II and III mean that Conditions (A) and (B) are sufficient for the Cauchy problem of $A$ to be E-well posed. 


\section{References}

[1] Oleinik, O. A., On the Cauchy problem for weakly hyperbolic equations, Comm. Pure Appl. Math., 23 (1970).

[2] Menikoff, A., The Cauchy problem for weakly hyperbolic equations, Amer. J. Math., 97 (1975).

[3] Ohya, Y., Le problème de Cauchy à caractéristiques multiples, C. R. Acad. Sc. Paris, 282 (1976).

[4] Svensson, S. L., Necessary and sufficient conditions for the hyperbolicity of polynomials with hyperbolic principal part, Arkiv för Mat., 8 (1969).

[5] Sakamoto, R., Mixed problems for hyperbolic equations I, II, J. Math. Kyoto Univ., 10 (1970). 